\title{
HOCHSCHILD COHOMOLOGY OF THE INTEGRAL GROUP RING OF THE DIHEDRAL GROUP. I: EVEN CASE
}

\author{
A. I. GENERALOV
}

Dedicated to the centenary of D. K. Faddeev's birth

\begin{abstract}
A free bimodule resolution is constructed for the integral group ring of the dihedral group of order $4 m$. This resolution is applied for a description, in terms of generators and defining relations, of the Hochschild cohomology algebra of this group ring.
\end{abstract}

\section{INTRODUCTION}

Let $K$ be a commutative ring with unity, let $R$ be an associative $K$-algebra that is a finitely generated projective $K$-module, let $\Lambda=R^{\mathrm{e}}=R \otimes_{K} R^{\text {op }}$ be its enveloping algebra, and let $\mathrm{HH}^{n}(R)=\operatorname{Ext}_{\Lambda}^{n}(R, R)$ be the $n$th Hochschild cohomology group of the algebra $R$ (with coefficients in the $R$-bimodule $R$ ). On the Abelian group

$$
\mathrm{HH}^{*}(R)=\bigoplus_{n \geq 0} \operatorname{HH}^{n}(R)=\bigoplus_{n \geq 0} \operatorname{Ext}_{\Lambda}^{n}(R, R),
$$

we introduce a structure of an associative algebra with the $\smile$-product (see [1, §5], [2, Chapter XI], and [3]); this algebra is called the Hochschild cohomology algebra. The algebra $\mathrm{HH}^{*}(R)$ is a graded commutative algebra, see [3]; moreover, it is well known (see, e.g., [4, p. 120]) that the $\smile$-product on $\mathrm{HH}^{*}(R)$ coincides with the Yoneda product.

In recent years, interest has grown in the investigation of the multiplicative structure of the Hochschild cohomology algebra, and appreciable success has been made in the solution of this problem for finite-dimensional algebras. In [5], a description was obtained of the Hochschild cohomology algebra for the symmetric group $S_{3}$ over the field $\mathbb{F}_{3}$ and for the alternating group $A_{4}$ and the dihedral 2-groups over the field $\mathbb{F}_{2}$. In $[6$, the algebra $\mathrm{HH}^{*}(R)$ was described in the case where $R$ is a self-injective Nakayama algebra. In the author's papers [7, 8, a description of the Hochschild cohomology algebra was given for algebras of dihedral type in the family $D(3 \mathcal{K})$ over an algebraically closed ground field of characteristic two and for a family of local algebras of quaternion type, respectively. We recall that algebras of dihedral, semidihedral, and quaternion types appeared in the work of K. Erdmann on classification of group blocks of tame representation type (see [9]). Some partial results were obtained in the description of the algebra $\operatorname{HH}^{*}(R)$ for the so-called Möbius algebra (see [10, 11) and for group blocks of tame representation type that have one or three simple modules (see [12]).

On the other hand, the Hochschild cohomology algebra was calculated recently in [13. for the integral group ring of the generalized quaternion group (see also [14]). It should be noted that a ring isomorphism $\mathrm{HH}^{*}(K[G]) \simeq \mathrm{H}^{*}(G) \otimes_{K} K[G]$, where $K$ is a commutative

2000 Mathematics Subject Classification. Primary 13D03.

Key words and phrases. Free bimodule resolution, integral group ring, dihedral group, Hochschild cohomology algebra.

Supported by RFBR (grant no. 06-01-0020/ 0). 
ring, was established in [15, 16]. But results in [13] and 7] show that the relationship between ordinary cohomology and Hochschild cohomology for noncommutative groups may turn out to be much more complicated.

In the present paper, a similar problem of description of the algebra $\mathrm{HH}^{*}\left(\mathbb{Z}\left[D_{4 m}\right]\right)$ is solved for the group ring $\mathbb{Z}\left[D_{4 m}\right]$ of the "even" dihedral proup of order $4 \mathrm{~m}$. Our approach to the calculation of products in $\mathrm{HH}^{*}(R)$ differs from that of the papers [5, 13, 14, where, in calculating the $\smile$-product in $\mathrm{HH}^{*}(R)$, the authors used reduction to the $\smile$-product in the usual cohomology of groups. Namely, we apply an approach that was used earlier in [7, 8. Its specific feature is that, on the basis of some empirical observations, a conjecture is stated about the structure of the bimodule projective resolution of the algebra $R$, and then, upon establishing this conjecture, the resolution is used in the calculation of the Hochschild cohomology algebra; in this way, we interpret the multiplication in $\mathrm{HH}^{*}(R)$ as the Yoneda product. We note that, for the first time, such a technique of calculating cohomology was used in [17, where the Yoneda algebra was described for two families of algebras of semidihedral type. The resulting description of the algebra $\mathrm{HH}^{*}\left(\mathbb{Z}\left[D_{4 m}\right]\right)$ can be used in the further investigation of the relationship between the Hochschild cohomology and the usual cohomology. Recall that the (usual) cohomology ring $\mathrm{H}^{*}\left(\mathbb{Z}\left[D_{2 t}\right]\right)$ for the dihedral group $D_{2 t}$ was calculated in [18].

We outline the structure of the paper. In $\S 1$, we state the main result of the paper, Theorem 1.1 , the algebra $\mathrm{HH}^{*}\left(\mathbb{Z}\left[D_{4 m}\right]\right)$ is determined in terms of generators and relations. In $\S 2$, we build some bicomplex and use the spectral sequence of the bicomplex to prove that the total complex of the bicomplex is a $\Lambda$-free resolution of the $\Lambda$-module $R=\mathbb{Z}\left[D_{4 m}\right]$; here $\Lambda=R^{\mathrm{e}}$. With the help of this resolution, in $\S 3$ we compute the groups $\mathrm{HH}^{n}\left(\mathbb{Z}\left[D_{4 m}\right]\right)$. Finally, in $\S 4$ we pick a (finite) set of generators for the algebra $\mathrm{HH}^{*}\left(\mathbb{Z}\left[D_{4 m}\right]\right)$ and find relations satisfied by these generators.

The author has found also a $\Lambda$-free bimodule resolution for the algebra $\mathbb{Z}\left[D_{4 m+2}\right]$ (the "odd case"). This resolution differs from that in the "even case". Because of this, the cohomology algebra $\mathrm{HH}^{*}\left(\mathbb{Z}\left[D_{4 m+2}\right]\right)$ is computed independently of the results of the present paper. These computations will be presented in the second part of this article.

\section{§1. Statement of the MAin RESUlt}

The dihedral group $D_{2 t}$ of order $2 t$ can be defined as a semidirect product of the cyclic group $C_{t}$ of order $t>2$ by the cyclic group $C_{2}$ :

$$
D_{2 t}=C_{t} \lambda C_{2}=\left\langle a, b \mid a^{t}=b^{2}=1, b a b=a^{-1}\right\rangle .
$$

In the sequel, we study only the case where $t$ is even: $t=2 m, m \geq 2$ (the "even case"). Then the dihedral group $D_{4 m}$ admits the following description (see, e.g., [19]):

$$
D_{4 m}=\left\langle b, c \mid b^{2}=c^{2}=1,(b c)^{m}=(c b)^{m}\right\rangle
$$

(to deduce (1.2) from (1.1), it suffices to put $c=a b$ ). Any element of $D_{4 m}$ different from 1 and $(b c)^{m}$ is represented uniquely (in the "alphabet" $\{b, c\}$ ) by one of the following "words":

$$
(b c)^{i},(c b)^{i}(1 \leq i \leq m-1) ; b(c b)^{i}, c(b c)^{i}(0 \leq i \leq m-1) .
$$

Such a representation of elements of $D_{4 m}$ is said to be standard. The empty word 1 will be called the standard representation of the unit element of the group; and the standard representation for the element $(b c)^{m}=(c b)^{m}$ is the word $(b c)^{m}$. For an element $x$ of the group algebra $\mathbb{Z}[G]$ with $G=D_{4 m}$, a presentation in the form $x=\sum_{g \in G} x_{g} g$, where $x_{g} \in$ $\mathbb{Z}$ and all elements $g \in G$ have standard representations, is called the canonical expansion. The canonical expansion of an element of the envelope algebra, $\lambda=\sum_{g, h \in G} \lambda(g, h) g \otimes h \in$ $\Lambda=R^{\mathrm{e}}$, is defined similarly ( $g$ and $h$ have the standard representations and $\lambda(g, h) \in \mathbb{Z}$ ). 
Consider the polynomials defined by the following formulas:

$$
f_{i}(T)=\left(\frac{T+\sqrt{T^{2}-4}}{2}\right)^{i}+\left(\frac{T-\sqrt{T^{2}-4}}{2}\right)^{i} \in \mathbb{Z}[T] \quad(i \in \mathbb{N}) .
$$

This family of polynomials can also be introduced with the help of the recurrence formulas

$$
f_{0}(T):=2, f_{1}(T):=T, f_{i+1}(T):=f_{i}(T) \cdot T-f_{i-1}(T) \text { for } i \geq 1 .
$$

Observe that, for $i \geq 1$, the $f_{i}$ are unital polynomials with $\operatorname{deg} f_{i}=i$, so that we can write

$$
f_{i}(T)=T^{i}+g_{i}(T)
$$

for some $g_{i} \in \mathbb{Z}[T], i \geq 1$, with $\operatorname{deg} g_{i}<i$.

Put $R_{m}=\mathbb{Z}\left[D_{4 m}\right]$. To describe the Hochschild cohomology algebra $\operatorname{HH}^{*}\left(R_{m}\right)$, we construct auxiliary graded $\mathbb{Z}$-algebras. Set

$$
\mathcal{X}=\left\{p_{1}, p_{1}^{\prime}, p_{2}, p_{3}, u_{0}, u_{1}, u_{1}^{\prime}, u_{2}, u_{2}^{\prime}, v, w\right\} .
$$

On the algebra $\mathbb{Z}[\mathcal{X}]$, we introduce a grading such that

$$
\begin{aligned}
& \operatorname{deg} p_{1}=\operatorname{deg} p_{1}^{\prime}=\operatorname{deg} p_{2}=\operatorname{deg} p_{3}=0, \\
& \operatorname{deg} u_{0}=\operatorname{deg} u_{1}=\operatorname{deg} u_{1}^{\prime}=\operatorname{deg} u_{2}=\operatorname{deg} u_{2}^{\prime}=2, \\
& \operatorname{deg} v=3, \operatorname{deg} w=4 .
\end{aligned}
$$

For brevity of the further statements, we denote

$$
q_{i}:=f_{i}\left(p_{2}\right) \in \mathbb{Z}[\mathcal{X}], \quad 1 \leq i \leq m .
$$

Then we define a graded $\mathbb{Z}$-algebra $\mathcal{A}_{m}=\mathbb{Z}[\mathcal{X}] / I_{m}$, where $I_{m}$ is the ideal of the algebra $\mathbb{Z}[\mathcal{X}]$ generated by the following elements:

— of degree 0:

$$
p_{1}^{2}-\left(p_{1}^{\prime}\right)^{2}, p_{1}^{2}-\varkappa_{1}
$$

with

$$
\begin{aligned}
& \varkappa_{1}= \begin{cases}m\left(1+\sum_{i=1}^{\left[\frac{m-1}{2}\right]} q_{2 i}\right) & \text { if } m \text { is odd, } \\
m\left(1+p_{3}+\sum_{i=1}^{\left[\frac{m-1}{2}\right]} q_{2 i}\right) & \text { if } m \text { is even; }\end{cases} \\
& p_{1} p_{1}^{\prime}-\varkappa_{2}
\end{aligned}
$$

with

$$
\begin{aligned}
& \varkappa_{2}= \begin{cases}m\left(p_{3}+\sum_{i=1}^{\left[\frac{m}{2}\right]} q_{2 i-1}\right) & \text { if } m \text { is odd, } \\
m \sum_{i=1}^{\left[\frac{m}{2}\right]} q_{2 i-1} & \text { if } m \text { is even; }\end{cases} \\
& p_{1} p_{3}-\varkappa_{3}, p_{1}^{\prime} p_{3}-\varkappa_{3}^{\prime}
\end{aligned}
$$

with

$$
\varkappa_{3}=\left\{\begin{array}{ll}
p_{1}^{\prime} & \text { if } m \text { is odd }, \\
p_{1} & \text { if } m \text { is even, }
\end{array} \quad \varkappa_{3}^{\prime}= \begin{cases}p_{1} & \text { if } m \text { is odd } \\
p_{1}^{\prime} & \text { if } m \text { is even }\end{cases}\right.
$$




$$
p_{1} p_{2}-2 p_{1}^{\prime}, p_{1}^{\prime} p_{2}-2 p_{1}, q_{m}-2 p_{3}, p_{2} p_{3}-q_{m-1}, p_{3}^{2}-1 \text {; }
$$

— of degree 2 :

$$
p_{1} u_{0}, p_{1}^{\prime} u_{0}, 2 m u_{0} ; 2 u_{i}, 2 u_{i}^{\prime} \text { for } i \in\{1,2\} \text {; }
$$
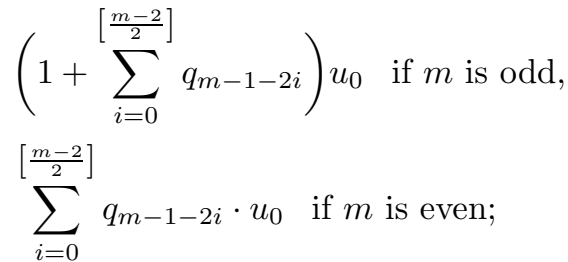

$$
p_{3} u_{0}+\varkappa_{4}
$$

with

$$
\begin{aligned}
& \varkappa_{4}= \begin{cases}\left(\sum_{i=0}^{\left[\frac{m-3}{2}\right]} q_{m-2-2 i}\right) u_{0} & \text { if } m \text { is odd, } \\
\left(1+\sum_{i=0}^{\left[\frac{m-3}{2}\right]} q_{m-2-2 i}\right) u_{0} & \text { if } m \text { is even; }\end{cases} \\
& p_{1} u_{1}-\varkappa_{5}, p_{1}^{\prime} u_{1}^{\prime}-\varkappa_{5}^{\prime}
\end{aligned}
$$

with

$$
\varkappa_{5}=\left\{\begin{array}{ll}
u_{2} & \text { if } m \text { is odd, } \\
u_{2}+p_{3} u_{2} & \text { if } m \text { is even, }
\end{array} \quad \varkappa_{5}^{\prime}= \begin{cases}u_{2}^{\prime} & \text { if } m \text { is odd, } \\
u_{2}^{\prime}+p_{3} u_{2}^{\prime} & \text { if } m \text { is even; }\end{cases}\right.
$$

with $p_{1}^{\prime} u_{1}-\varkappa_{6}, \quad p_{1} u_{1}^{\prime}-\varkappa_{6}^{\prime}$

with

$$
\begin{aligned}
& \varkappa_{7}= \begin{cases}u_{1}+m\left(\left[\frac{m}{2}\right] \cdot 1+\sum_{i=1}^{\left[\frac{m-2}{2}\right]}\left(\left[\frac{m}{2}\right]-i\right) q_{2 i}\right) u_{0} & \text { if } m \text { is odd, } \\
u_{1}+p_{3} u_{1}+m\left(\sum_{i=1}^{\left[\frac{m-2}{2}\right]}\left(\left[\frac{m}{2}\right]-i\right) q_{2 i-1}\right) u_{0} & \text { if } m \text { is even; }\end{cases} \\
& \varkappa_{7}^{\prime}= \begin{cases}u_{1}^{\prime}+m\left(\left[\frac{m}{2}\right] \cdot 1+\sum_{i=1}^{\left[\frac{m-2}{2}\right]}\left(\left[\frac{m}{2}\right]-i\right) q_{2 i}\right) u_{0} & \text { if } m \text { is odd, } \\
u_{1}^{\prime}+p_{3} u_{1}^{\prime}+m\left(\sum_{i=1}^{\left[\frac{m-2}{2}\right]}\left(\left[\frac{m}{2}\right]-i\right) q_{2 i-1}\right) u_{0} & \text { if } m \text { is even; }\end{cases} \\
& p_{1}^{\prime} u_{2}-\varkappa_{8}, \quad p_{1} u_{2}^{\prime}-\varkappa_{8}^{\prime}
\end{aligned}
$$


with

$$
\begin{aligned}
& \varkappa_{8}= \begin{cases}p_{3} u_{1}+m\left(\sum_{i=0}^{\left[\frac{m-2}{2}\right]}\left(\left[\frac{m}{2}\right]-i\right) q_{2 i+1}\right) u_{0} & \text { if } m \text { is odd, } \\
m\left(\left[\frac{m}{2}\right] \cdot 1+\sum_{i=1}^{\left[\frac{m-2}{2}\right]}\left(\left[\frac{m}{2}\right]-i\right) q_{2 i}\right) u_{0} & \text { if } m \text { is even; }\end{cases} \\
& \varkappa_{8}^{\prime}= \begin{cases}p_{3} u_{1}^{\prime}+m\left(\sum_{i=0}^{\left[\frac{m-2}{2}\right]}\left(\left[\frac{m}{2}\right]-i\right) q_{2 i+1}\right) u_{0} & \text { if } m \text { is odd }, \\
m\left(\left[\frac{m}{2}\right] \cdot 1+\sum_{i=1}^{\left[\frac{m-2}{2}\right]}\left(\left[\frac{m}{2}\right]-i\right) q_{2 i}\right) u_{0} & \text { if } m \text { is even; }\end{cases}
\end{aligned}
$$

— of degree 3 :

$$
2 v, p_{2} v
$$

- of degree 4:

$2 m w, 2 p_{1} w, 2 p_{1}^{\prime} w, u_{0} u_{2}, u_{0} u_{2}^{\prime}, u_{0}^{2}-\left(2-q_{2}\right) w ;$

$$
u_{0} u_{1}-u_{0} u_{1}^{\prime}, u_{0} u_{1}-m q_{m-1} w, u_{1} u_{1}^{\prime}-\varkappa_{9}
$$

with

$$
\begin{aligned}
& \varkappa_{9}= \begin{cases}m w & \text { if } m \text { is odd } \\
0 & \text { if } m \text { is even; }\end{cases} \\
& u_{1} u_{2}^{\prime}-\varkappa_{10}, \quad u_{1}^{\prime} u_{2}-\varkappa_{10}^{\prime}
\end{aligned}
$$

with

$$
\begin{aligned}
& \varkappa_{10}=\left\{\begin{array}{ll}
p_{1}^{\prime} w & \text { if } m \text { is odd, } \\
0 & \text { if } m \text { is even, }
\end{array} \quad \varkappa_{10}^{\prime}= \begin{cases}p_{1} w & \text { if } m \text { is odd } \\
0 & \text { if } m \text { is even; }\end{cases} \right. \\
& u_{2}^{2}-u_{1}^{2}-\left(u_{2}^{\prime}\right)^{2}+\left(u_{1}^{\prime}\right)^{2}, \quad u_{2}^{2}-u_{1}^{2}-\varkappa_{11}
\end{aligned}
$$

with

$$
\varkappa_{11}= \begin{cases}m \sum_{i=1}^{\left[\frac{m-1}{2}\right]} q_{2 i} \cdot w & \text { if } m \text { is odd, } \\ m\left(1+p_{3}+\sum_{i=1}^{\left[\frac{m-1}{2}\right]} q_{2 i}\right) w & \text { if } m \text { is even; }\end{cases}
$$

with

$$
\varkappa_{12}= \begin{cases}m\left(p_{3}+\sum_{i=1}^{\left[\frac{m-1}{2}\right]} q_{2 i-1}\right) w & \text { if } m \text { is odd, } \\ m \sum_{i=0}^{\left[\frac{m-1}{2}\right]} q_{2 i+1} \cdot w & \text { if } m \text { is even; }\end{cases}
$$

— of degree 5 :

$$
u_{0} v
$$


— of degree 6 :

$$
v^{2}-\left(u_{1}+u_{1}^{\prime}\right) w .
$$

Moreover, we induce a grading on the algebra $\mathcal{A}_{m}$ from the grading on $\mathbb{Z}[\mathcal{X}]$.

Theorem 1.1. Let $R=\mathbb{Z}\left[D_{4 m}\right]$, where $m \in \mathbb{N} \backslash\{1\}$. The Hochschild cohomology algebra $\mathrm{HH}^{*}(R)$ is isomorphic, as a graded algebra, to the algebra $\mathcal{A}_{m}$.

Remark 1.2. We observe that the above description of the algebra $\mathrm{HH}^{*}\left(\mathbb{Z}\left[D_{4 m}\right]\right)$ implies immediately that this algebra is commutative.

Remark 1.3. The defining relations (1.6)-(1.26) of the algebra $\mathcal{A}_{m}$ imply that its description can be simplified with regard to the parity of $m$ (for example, if $m$ is odd, then in the generating set $\mathcal{X}$ we can omit the elements $\left.p_{1}^{\prime}, u_{2}, u_{2}^{\prime}\right)$. The above statement of Theorem 1.1 is convenient for unifying the arguments in the proofs. But at the end of the paper, for the reader's convenience, we state Corollaries 4.11 and 4.12 in which we take into account the possibilities of simplifying the description of the cohomology algebra $\mathrm{HH}^{*}\left(\mathbb{Z}\left[D_{4 m}\right]\right)$.

In the course of the proof of Theorem 1.1 we compute the groups $\mathrm{HH}^{n}(R)$. This is of independent interest, and we collect the results in the following statement.

Proposition 1.4. Let $R=\mathbb{Z}\left[D_{4 m}\right]$. Then

$$
\mathrm{HH}^{n}(R)= \begin{cases}\mathbb{Z}^{m+3} & \text { if } n=0 \\ \mathbb{Z}_{2 m}^{m+1} \oplus \mathbb{Z}_{2}^{8 k+2} & \text { if } n=4 k>0, \\ \mathbb{Z}_{2}^{8 k} & \text { if } n=4 k+1 \\ \mathbb{Z}_{2 m}^{m-1} \oplus \mathbb{Z}_{2}^{8(k+1)} & \text { if } n=4 k+2 \\ \mathbb{Z}_{2}^{8 k+4} & \text { if } n=4 k+3\end{cases}
$$

\section{$\S 2$. Resolution}

Put $G=D_{4 m}$ and $R=R_{m}=\mathbb{Z}[G]$. We consider the following elements of the algebra $\Lambda=R^{\mathrm{e}}$ :

$$
\begin{gathered}
B^{+}=b \otimes 1+1 \otimes b, C^{+}=c \otimes 1+1 \otimes c, \\
B^{-}=b \otimes 1-1 \otimes b, C^{-}=c \otimes 1-1 \otimes c, \\
\zeta^{+}=\sum_{i=0}^{m-1} c(b c)^{i} \otimes(c b)^{m-1-i}+\sum_{i=0}^{m-1}(b c)^{i} \otimes(c b)^{m-1-i} c, \\
\eta^{+}=\sum_{i=0}^{m-1} b(c b)^{i} \otimes(b c)^{m-1-i}+\sum_{i=0}^{m-1}(c b)^{i} \otimes(b c)^{m-1-i} b, \\
\zeta^{-}=\sum_{i=0}^{m-1} c(b c)^{i} \otimes(c b)^{m-1-i}-\sum_{i=0}^{m-1}(b c)^{i} \otimes(c b)^{m-1-i} c, \\
\eta^{-}=\sum_{i=0}^{m-1} b(c b)^{i} \otimes(b c)^{m-1-i}-\sum_{i=0}^{m-1}(c b)^{i} \otimes(b c)^{m-1-i} b, \\
\rho^{+}=c(b c)^{m-1} \otimes 1+1 \otimes(c b)^{m-1} c, \omega^{+}=b(c b)^{m-1} \otimes 1+1 \otimes(b c)^{m-1} b, \\
\rho^{-}=c(b c)^{m-1} \otimes 1-1 \otimes(c b)^{m-1} c, \omega^{-}=b(c b)^{m-1} \otimes 1-1 \otimes(b c)^{m-1} b .
\end{gathered}
$$


In the category of (left) $\Lambda$-modules, we construct the following bicomplex, lying in the first quadrant of the plane (i.e., its rows and columns are enumerated by $0,1,2, \ldots$ ):

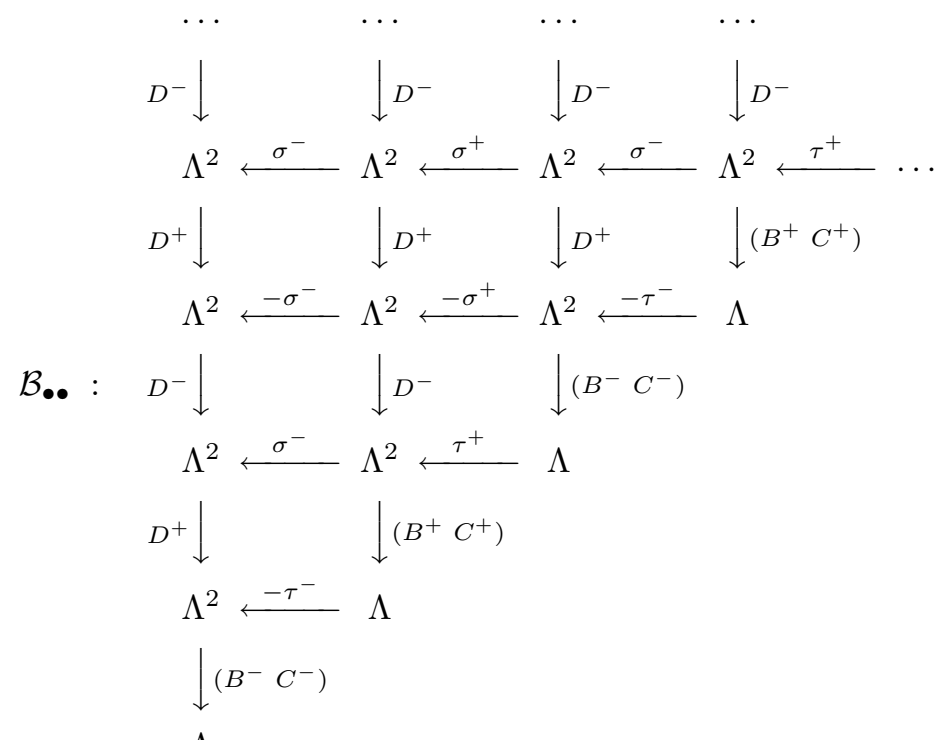

$\Lambda$

where

$$
\begin{gathered}
D^{+}=\left(\begin{array}{cc}
B^{+} & 0 \\
0 & C^{+}
\end{array}\right), \quad D^{-}=\left(\begin{array}{cc}
B^{-} & 0 \\
0 & C^{-}
\end{array}\right), \quad \tau^{+}=\left(\begin{array}{c}
\zeta^{+} \\
-\eta^{+}
\end{array}\right), \quad \tau^{-}=\left(\begin{array}{c}
\zeta^{-} \\
-\eta^{-}
\end{array}\right), \\
\sigma^{+}=\left(\begin{array}{cc}
\rho^{+} & 0 \\
0 & -\omega^{+}
\end{array}\right), \quad \sigma^{-}=\left(\begin{array}{cc}
\rho^{-} & 0 \\
0 & -\omega^{-}
\end{array}\right) .
\end{gathered}
$$

Theorem 2.1. The total complex $Q_{\bullet}=\operatorname{Tot}\left(\mathcal{B}_{\bullet \bullet}\right)$ of the bicomplex $(2.3)$ is a free resolution of the $\Lambda$-module $R$.

Remark 2.2. A direct verification shows that diagram (2.3) indeed represents a bicomplex; i.e., its rows and columns are differential sequences and all squares are anticommutative.

Remark 2.3. As usual, as an augmentation map $\mu: Q_{0}=\Lambda \rightarrow R$ we take the canonical map induced by multiplication on $R: \mu(x \otimes y)=x y$. Then the exactness of the corresponding sequence at the member $Q_{0}$, i.e., the fact that $\operatorname{Im} d_{0}^{Q}=\operatorname{Ker} \mu$, is well known.

Lemma 2.4. Let $B^{ \pm}, C^{ \pm}: \Lambda \rightarrow \Lambda$ be homomorphisms of multiplication (on the right) by the corresponding elements (see (2.1), (2.2)). Then

$$
\begin{array}{ll}
\operatorname{Ker} B^{-}=\operatorname{Im} B^{+}, & \operatorname{Ker} B^{+}=\operatorname{Im} B^{-}, \\
\operatorname{Ker} C^{-}=\operatorname{Im} C^{+}, & \operatorname{Ker} C^{+}=\operatorname{Im} C^{-} .
\end{array}
$$

Proof. We only prove the first relation in (2.6). The remaining relations can be proved similarly (or can be derived from the first by symmetry).

Let $\lambda \in \operatorname{Ker} B^{-}$, and let $\lambda=\sum_{u, v \in G} \lambda(u, v) u \otimes v$ be the canonical expansion of this element. Since

$$
\lambda \cdot b \otimes 1=\sum_{u, v \in G} \lambda\left(u b^{-1}, v\right) u \otimes v, \quad \lambda \cdot 1 \otimes b=\sum_{u, v \in G} \lambda\left(u, b^{-1} v\right) u \otimes v,
$$


we have $\lambda(u b, v)=\lambda(u, b v)$ for all $u, v \in G$. Consequently,

$$
\lambda=\sum_{u, v} \lambda(u b, v)(u b \otimes v+u \otimes b v)=\sum_{u, v} \lambda(u b, v) u \otimes v \cdot(b \otimes 1+1 \otimes b) \in \operatorname{Im} B^{+} .
$$

Lemma 2.5. $\operatorname{Ker} d_{0}^{Q}=\operatorname{Im} d_{1}^{Q}$.

Proof. For brevity, we denote $d_{i}=d_{i}^{Q}$. It remains to prove the inclusion Ker $d_{0} \subset \operatorname{Im} d_{1}$. Let $h_{1}, h_{2}, h_{3}$ denote the columns of the matrix of the differential $d_{1}$,

$$
d_{1}=\left(\begin{array}{ccc}
-\zeta^{-} & B^{+} & 0 \\
\eta^{-} & 0 & C^{+}
\end{array}\right),
$$

which are also viewed as elements in $\operatorname{Im} d_{1} \subset \Lambda^{2}$. Suppose that $q=(\lambda, \mu) \in \operatorname{Ker} d_{0}$ with $\lambda, \mu \in \Lambda$. Let $\lambda=\sum_{u, v \in G} \lambda(u, v) u \otimes v$ and $\mu=\sum_{u, v \in G} \mu(u, v) u \otimes v$ be canonical expansions, where $\lambda(u, v), \mu(u, v) \in \mathbb{Z}$. Since

$$
\lambda \cdot B^{-}+\mu \cdot C^{-}=0,
$$

we have

$$
\lambda(u b, v)-\lambda(u, b v)+\mu(u c, v)-\mu(u, c v)=0
$$

for all $u, v \in G$. Indeed, the left-hand side of equation (2.8) is the coefficient of $u \otimes v$ in the expansion of the element $\lambda \cdot B^{-}+\mu \cdot C^{-}$in the canonical basis of the algebra $\Lambda$. If the standard representation of an element $v \in G$ starts with the letter $c$ or if $v=1$, then we may assume that $\lambda(u, b v)=0$ for all $u$. Indeed, if $\lambda(u, b v) \neq 0$, then we replace the original $q \in \operatorname{Ker} d_{0}$ by the element $q^{\prime}=q-\lambda(u, b v) u \otimes v \cdot h_{2}$. By symmetry, we may also assume that $\mu(u, c v)=0$ if the standard representation for an element $v \in G$ starts with $b$ (or if $v=1$ ). We fix $u \in G$ and put $\mu_{u}:=\mu(u c, 1)$. From (2.8), it follows that

$$
\begin{aligned}
\mu\left(u(c b)^{i} c,(b c)^{i}\right) & =\lambda\left(u(c b)^{i+1}, c(b c)^{i}\right) \text { for } 0 \leq i \leq m-1, \\
\lambda\left(u(c b)^{i}, c(b c)^{i-1}\right) & =\mu\left(u(c b)^{i} c,(b c)^{i}\right) \text { for } 1 \leq i \leq m-1,
\end{aligned}
$$

whence

$$
\begin{aligned}
\mu\left(u(c b)^{i} c,(b c)^{i}\right) & =\mu_{u} \text { for } 1 \leq i \leq m-1, \\
\lambda\left(u(c b)^{i}, c(b c)^{i-1}\right) & =\mu_{u} \text { for } 1 \leq i \leq m .
\end{aligned}
$$

Furthermore, putting $v=1$ and $v=(b c)^{m}$ in (2.8), we obtain two additional relations:

$$
\lambda(u b, 1)+\mu(u c, 1)=0, \quad \lambda\left(u(b c)^{m}, c(b c)^{m-1}\right)+\mu\left(u(b c)^{m}, b(c b)^{m-1}\right)=0 .
$$

By symmetry, we have

$$
\begin{aligned}
\lambda\left(u(b c)^{i} b,(c b)^{i}\right) & =\mu\left(u(b c)^{i+1}, b(c b)^{i}\right) \text { for } 0 \leq i \leq m-1, \\
\mu\left(u(b c)^{i}, b(c b)^{i-1}\right) & =\lambda\left(u(b c)^{i} b,(c b)^{i}\right) \text { for } 1 \leq i \leq m-1,
\end{aligned}
$$

whence

$$
\begin{aligned}
\lambda\left(u(b c)^{i} b,(c b)^{i}\right) & =-\mu_{u} \text { for } \quad 0 \leq i \leq m-1, \\
\mu\left(u(b c)^{i}, b(c b)^{i-1}\right) & =-\mu_{u} \text { for } \quad 1 \leq i \leq m-1 .
\end{aligned}
$$

These relations imply easily that

$$
q=\left(\sum_{u} \mu_{u} u\right)(b c)^{m} \otimes 1 \cdot h_{1} \in \operatorname{Im} d_{1} .
$$

Lemma 2.6. a) $\operatorname{Ker}\left(\zeta^{-} B^{-}\right)^{*}=\operatorname{Im}\left(B^{+}\right)^{*}+\operatorname{Im}\left(C^{+}\right)^{*}$;

b) $\operatorname{Ker}\left(\zeta^{+} B^{+}\right)^{*}=\operatorname{Im}\left(B^{-}\right)^{*}+\operatorname{Im}\left(C^{-}\right)^{*}$. 
Proof. a) Using Remark 2.2, we deduce the inclusion $\operatorname{Im}\left(B^{+}\right)^{*}+\operatorname{Im}\left(C^{+}\right)^{*} \subset \operatorname{Ker}\left(\zeta^{-} B^{-}\right)^{*}$ from the fact that

$$
B^{+} \cdot \zeta^{-} B^{-}=B^{+} \eta^{-} C^{-}=0, C^{+} \cdot \zeta^{-} B^{-}=0
$$

in the algebra $\Lambda$. Now we prove the reverse inclusion. Let $\lambda=\sum_{u, v \in G} \lambda(u, v) u \otimes v \in$ $\operatorname{Ker}\left(\zeta^{-} B^{-}\right)^{*}$ be a nonzero element, where $\lambda(u, v) \in \mathbb{Z}$. Consider the set

$$
V_{\lambda}=\{v \in G \mid \lambda(u, v) \neq 0 \text { for some } u\}
$$

and put $l(\lambda):=\max \left\{l(v) \mid v \in V_{\lambda}\right\}$. Also, we consider the set $M_{\lambda}=\left\{v \in V_{\lambda} \mid l(v)=\right.$ $l(\lambda)\}$. Since

$$
\begin{aligned}
\zeta^{-} B^{-}=\sum_{i=0}^{m-1}\left((c b)^{i+1} \otimes(c b)^{m-1-i}\right. & -(b c)^{i} b \otimes(c b)^{m-1-i} c \\
& \left.-c(b c)^{i} \otimes b(c b)^{m-1-i}+(b c)^{i} \otimes(b c)^{m-i}\right),
\end{aligned}
$$

we have

$$
\begin{aligned}
& \sum_{i=0}^{m-1}\left(\lambda\left(u \cdot(b c)^{i+1},(b c)^{m-1-i} \cdot v\right)-\lambda\left(u \cdot(b c)^{i} b,(c b)^{m-1-i} c \cdot v\right)\right. \\
& \left.-\lambda\left(u \cdot c(b c)^{i},(b c)^{m-1-i} b \cdot v\right)+\lambda\left(u \cdot(c b)^{i},(c b)^{m-i} \cdot v\right)\right)=0
\end{aligned}
$$

for all $u, v \in G$. Fix $v \in M_{\lambda}$. First, we assume that the standard representation of $v$ starts with $b$ and write $v=b v_{1}$, where either $v_{1}=1$, or the standard representation of $v_{1}$ starts with $c$. Replacing the initial element $\lambda$ by the element $\lambda^{\prime}:=\lambda-\lambda(u, v) u \otimes v_{1} \cdot B^{+}$, we obtain a new element with zero coefficient of $u \otimes v$ in its canonical expansion. Now, if the standard representation of $v$ starts with the letter $c$ and $v=c v_{2}$, where either $v_{2}=1$ or the standard representation of $v_{2}$ starts with $b$, then we replace $\lambda$ by $\lambda^{\prime \prime}:=$ $\lambda-\lambda(u, v) u \otimes v_{2} \cdot C^{+}$. Applying a similar argument for each $u \in G$ with $\lambda(u, v) \neq 0$, we obtain an element $\lambda_{1} \in \operatorname{Ker}\left(\zeta^{-} B^{-}\right)^{*}$ for which the cardinality of the set $M_{\lambda_{1}}$ is less than that of $M_{\lambda}$. Repeating this procedure, we obtain an element $\tilde{\lambda}$ with $l(\widetilde{\lambda})<l(\lambda)$.

The above process terminates at an element of $\operatorname{Ker}\left(\zeta^{-} B^{-}\right)^{*}$ whose canonical expansion can have nonzero coefficients only at basis elements of the form $u \otimes 1, u \in G$. Hence, we may assume that the initial element $\lambda \in \operatorname{Ker}\left(\zeta^{-} B^{-}\right)^{*}$ has the property that $\lambda(u, v)=0$ for all $v \in G \backslash\{1\}$ and $u \in G$.

Observe that, for a fixed $v \in G$, the set $G$ is a disjoint union of the sets

$$
\left\{(b c)^{m-1-i} \cdot v\right\}_{i=0}^{m-1},\left\{(c b)^{m-1-i} c \cdot v\right\}_{i=0}^{m-1},\left\{(b c)^{m-1-i} b \cdot v\right\}_{i=0}^{m-1},\left\{(c b)^{m-i} \cdot v\right\}_{i=0}^{m-1} .
$$

By the above assumptions, only one summand remains on the left-hand side of (2.9), and this summand must also be zero. Since $G$ is a disjoint union of the sets

$$
\left\{u \cdot(b c)^{i+1}\right\}_{i=0}^{m-1}, \quad\left\{u \cdot(b c)^{i} b\right\}_{i=0}^{m-1}, \quad\left\{u \cdot(c b)^{i} c\right\}_{i=0}^{m-1}, \quad\left\{u \cdot(c b)^{i}\right\}_{i=0}^{m-1},
$$

we see that $\lambda(u, v)=0$ for all $u, v \in G$. This completes the proof of the inclusion $\operatorname{Ker}\left(\zeta^{-} B^{-}\right)^{*} \subset \operatorname{Im}\left(B^{+}\right)^{*}+\operatorname{Im}\left(C^{+}\right)^{*}$.

b) The proof is similar to that of part a).

Proof of Theorem 2.1. We consider the spectral sequence of the bicomplex $B_{\bullet \bullet}$ (see $(2.3)$ ),

$$
\mathrm{H}_{p}^{h} \mathrm{H}_{q}^{v}\left(\mathcal{B}_{\bullet \bullet}\right) \quad \Rightarrow \quad \mathrm{H}_{p+q}\left(\operatorname{Tot}\left(\mathcal{B}_{\bullet \bullet}\right)\right)
$$

and prove that the second sheet of this spectral sequence degenerates. 
By Lemma 2.4, the (nonzero) columns in $\mathcal{B}_{\text {.. }}$ are exact at all terms except the two bottom terms $\Lambda^{2}$ and $\Lambda$. Consequently, every row with number $i>0$ of the first sheet of the spectral sequence (2.10) contains two nonzero terms, namely,

$$
\operatorname{Ker}\left(B^{-}, C^{-}\right) / \operatorname{Im} D^{+} \stackrel{\overline{\tau^{-}}}{\longleftarrow} \operatorname{Coker}\left(B^{+}, C^{+}\right)
$$

for odd $i$ and

$$
\operatorname{Ker}\left(B^{+}, C^{+}\right) / \operatorname{Im} D^{-} \stackrel{\overline{\tau^{+}}}{\longleftarrow} \operatorname{Coker}\left(B^{-}, C^{-}\right)
$$

for even $i$; here $\overline{\tau^{-}}$and $\overline{\tau^{+}}$denote the homomorphisms induced by the homomorphisms $\tau^{-}$and $\tau^{+}$, respectively. We shall prove that $\overline{\tau^{-}}$is an isomorphism; the fact that $\overline{\tau^{+}}$is also an isomorphism is established similarly.

The surjectivity of $\overline{\tau^{-}}$follows from Lemma 2.5. Next, we assume that $\overline{\tau^{-}}(\bar{\lambda})=0$ for some $\bar{\lambda}=\lambda+\operatorname{Im}\left(B^{+}, C^{+}\right) \in \operatorname{Coker}\left(B^{+}, C^{+}\right)$, i.e., $\tau^{-}(\lambda)=D^{+}\left(\lambda^{\prime}, \lambda^{\prime \prime}\right)$ for some $\lambda^{\prime}, \lambda^{\prime \prime} \in \Lambda$. The latter relation is equivalent to the system

$$
\left\{\begin{aligned}
-\lambda \cdot \zeta^{-} & =\lambda^{\prime} \cdot B^{+}, \\
\lambda \cdot \eta^{-} & =\lambda^{\prime \prime} \cdot C^{+} .
\end{aligned}\right.
$$

Consequently, we have $\lambda \cdot \zeta^{-} B^{-}=0$. By Lemma 2.6 a), there exist $\mu^{\prime}, \mu^{\prime \prime} \in \Lambda$ such that $\lambda=\mu^{\prime} \cdot B^{+}+\mu^{\prime \prime} \cdot C^{+}$, i.e., $\lambda \in \operatorname{Im}\left(B^{+}, C^{+}\right)$, whence $\bar{\lambda}=0$.

Finally, in the 0th row of the first sheet of the spectral sequence, there is only one nonzero term: $\operatorname{Coker}\left(B^{-}, C^{-}\right) \simeq R$.

Thus, we have proved that $Q_{\bullet}=\operatorname{Tot}\left(\mathcal{B}_{\bullet \bullet}\right)$ is a $\Lambda$-free resolution of the module $R$.

Consider the bicomplex $\mathcal{A}_{\text {.• }}$ that consists of the first two columns of the bicomplex $\mathcal{B}_{\bullet \bullet}$ with numbers 0 and 1 (the remaining columns in $\mathcal{A}_{\bullet \bullet}$ are zero). Set $X_{\bullet}=\operatorname{Tot}\left(\mathcal{A}_{\bullet \bullet}\right)$.

Proposition 2.7. There is a short exact sequence of complexes

$$
0 \rightarrow X_{\bullet} \stackrel{i}{\longrightarrow} Q \bullet \stackrel{\pi}{\longrightarrow} Q \bullet[-4] \rightarrow 0
$$

that splits in each degree.

Proof. This follows immediately from the construction of the bicomplex $\mathcal{B}$...

\section{§3. Additive structure of the cohomology algebra}

We keep the notation $R$ for the group algebra $\mathbb{Z}[G]$ with $G=D_{4 m}$. In this section, we use the $\Lambda$-free resolution $Q_{\bullet}=\left(Q_{n}, d_{n}^{Q}\right)$ of the $\Lambda$-module $R$ (see Theorem 2.1) to determine the cohomology groups $\mathrm{HH}^{i}(R), i \geq 0$. For this, we investigate the complex

$$
\operatorname{Hom}_{\Lambda}(Q \bullet, R)=\left(\operatorname{Hom}_{\Lambda}\left(Q_{n}, R\right), \delta^{n}:=\left(d_{n}^{Q}\right)^{*}\right)
$$

and calculate its cohomology:

$$
\mathrm{H}^{n}\left(\operatorname{Hom}_{\Lambda}\left(Q_{\bullet}, R\right)\right)=\operatorname{Ext}_{\Lambda}^{n}(R, R)=\operatorname{HH}^{n}(R) .
$$

To describe the homomorphisms in $\operatorname{Hom}_{\Lambda}\left(Q_{n}, R\right)$, we fix the decomposition

$$
Q_{n}=\sum_{i+j=n} B_{i j}
$$

ordered in accordance with the increase of the second index $j$.

Since $Q_{n} \simeq \Lambda^{n+1}$, we have $\operatorname{Hom}_{\Lambda}\left(Q_{n}, R\right) \simeq R^{n+1}$, and any $\Lambda$-homomorphism $f: Q_{n} \rightarrow$ $R$ is identified with the collection $\left(f\left(e_{1}\right), f\left(e_{2}\right), \ldots, f\left(e_{n+1}\right)\right)$ of its values, where $e_{1}=$ $(1 \otimes 1,0, \ldots, 0), \ldots, e_{n+1}=(0, \ldots, 0,1 \otimes 1)$ is the canonical basis of the free $\Lambda$-module $\Lambda^{n+1}$. In the sequel, if in this collection there is a subsequence containing only zeros, say $r$ zeros, we denote such a subsequence by $\mathrm{O}_{r}$. Similarly, the zero $(r \times s)$-matrix will be 
denoted by $\mathrm{O}_{r, s}$; moreover, we omit the indices indicating the size of such a matrix if no confusion is possible.

We observe that, for the homomorphism $f=w^{*}: \Lambda \rightarrow \Lambda$ of multiplication on the right by $w \in \Lambda$, the induced homomorphism (of Abelian groups)

$$
\operatorname{Hom}_{\Lambda}(f, R): \operatorname{Hom}_{\Lambda}(\Lambda, R) \simeq R \rightarrow \operatorname{Hom}_{\Lambda}(\Lambda, R) \simeq R
$$

acts, in accordance with the identification mentioned above, as follows: $r \in R$ is mapped to $w * r$; here $*$ corresponds to the $\Lambda$-module structure on $R$.

Then the differential $\delta^{0}: R \rightarrow R^{2}$ can be described by the formula

$$
\delta^{0}(r)=\left(B^{-} * r, C^{-} * r\right)=(b r-r b, c r-r c)
$$

for any $r \in R$.

We consider the following elements of the algebra $R$ :

$$
\begin{aligned}
& \theta_{b}:=\sum_{i=0}^{\left[\frac{m-1}{2}\right]} b(c b)^{2 i}+\sum_{i=1}^{\left[\frac{m}{2}\right]} c(b c)^{2 i-1}, \\
& \theta_{c}:=\sum_{i=0}^{\left[\frac{m-1}{2}\right]} c(b c)^{2 i}+\sum_{i=1}^{\left[\frac{m}{2}\right]} b(c b)^{2 i-1} .
\end{aligned}
$$

It is clear that $\theta_{b}$ (respectively, $\theta_{c}$ ) is the sum of all elements of the group $G$ that are conjugate to $b$ (respectively, $c$ ).

Denote by $Z(R)$ the centre of the algebra $R$. The following statement is a consequence of well-known properties of group algebras (also it can be deduced directly from the analysis of the relation $\delta^{0}(r)=0$ with the help of $(\underline{3.2})$ ).

Proposition 3.1. The group $\operatorname{HH}^{0}(R) \simeq Z(R)$ is a free $\mathbb{Z}$-module with the basis

$$
\left\{1, \theta_{b}, \theta_{c},(b c)^{m}\right\} \cup\left\{(b c)^{i}+(c b)^{i}\right\}_{i=1}^{m-1} .
$$

Corollary 3.2. The group $\operatorname{Im} \delta^{0}$ is a free $\mathbb{Z}$-module of rank $3 m-3$.

Proof. Since $\operatorname{Im} \delta^{0} \subset R^{2}, \operatorname{Im} \delta^{0}$ is a free $\mathbb{Z}$-module, and we obtain

$$
\operatorname{rk} \operatorname{Im} \delta^{0}=\operatorname{rk} R-\operatorname{rk} \operatorname{Ker} \delta^{0}=4 m-(m+3)=3 m-3 .
$$

Remark 3.3. It is easy to verify that the following set is a basis of the $\mathbb{Z}$-module $\operatorname{Im} \delta^{0}$ :

$$
\begin{aligned}
\left\{\left((b c)^{i}-(c b)^{i}, 0\right)\right\}_{i=1}^{m-1} & \cup\left\{\left(0,(c b)^{i}-(b c)^{i}\right)\right\}_{i=1}^{m-1} \\
& \cup\left\{\left(c(b c)^{i-1}-b(c b)^{i}, c(b c)^{i}-b(c b)^{i-1}\right)\right\}_{i=1}^{m-1}
\end{aligned}
$$

The following properties of the elements $\theta_{b}$ and $\theta_{c}$ will be useful in the sequel.

Proposition 3.4. $\theta_{b}=\sum_{i=0}^{m-1}(b c)^{2 i} b, \theta_{c}=\sum_{i=0}^{m-1}(c b)^{2 i} c$.

Proof. We establish only the first relation; the second follows by symmetry. Replacing the index in the second sum in (3.3) by $j=m-i$, we obtain

$$
\sum_{i=1}^{\left[\frac{m}{2}\right]} c(b c)^{2 i-1}=\sum_{j=\left[\frac{m+1}{2}\right]}^{m-1} c(b c)^{2 m-2 j-1}=\sum_{j=\left[\frac{m+1}{2}\right]}^{m-1} c(c b)^{2 j+1}=\sum_{j=\left[\frac{m+1}{2}\right]}^{m-1} b(c b)^{2 j},
$$

whence

$$
\theta_{b}=\sum_{i=0}^{\left[\frac{m-1}{2}\right]} b(c b)^{2 i}+\sum_{i=\left[\frac{m+1}{2}\right]}^{m-1} b(c b)^{2 i}=\sum_{i=0}^{m-1} b(c b)^{2 i}
$$


Corollary 3.5. $b c \cdot \theta_{b}=\theta_{c}=c b \cdot \theta_{b}, \quad b c \cdot \theta_{c}=\theta_{b}=c b \cdot \theta_{c}$.

Proof. Proposition 3.4 yields

$$
b c \theta_{b}=b\left(\sum_{i=0}^{m-1} b(c b)^{2 i}\right) c=\sum_{i=0}^{m-1}(c b)^{2 i} c=\theta_{c} .
$$

The remaining relations are proved similarly.

Corollary 3.6. For any natural $j$, we have

$$
\sum_{i=0}^{m-1}(b c)^{2 i+2 j}=\sum_{i=0}^{m-1}(b c)^{2 i} .
$$

Proof. Multiplying both sides of the identity $\theta_{c} \cdot(b c)^{2 j}=\theta_{c}$ on the left by $c$, we arrive at (3.6).

Using the form of the differential $d_{1}^{Q}$ (see (2.7)), we see that the membership relation $\left(r_{1}, r_{2}\right) \in \operatorname{Ker} \delta^{1}$ is equivalent to the fact that the following system of three equations (over $R$ ) is satisfied:

$$
\begin{gathered}
-\zeta^{-} * r_{1}+\eta^{-} * r_{2}=0, \\
B^{+} * r_{1}=0, \\
C^{+} * r_{2}=0,
\end{gathered}
$$

or, in detail,

$$
\begin{gathered}
-\sum_{i=0}^{m-1} c(b c)^{i} \cdot r_{1} \cdot(c b)^{m-1-i}+\sum_{i=0}^{m-1}(b c)^{i} \cdot r_{1} \cdot(c b)^{m-1-i} c \\
+\sum_{i=0}^{m-1} b(c b)^{i} \cdot r_{2} \cdot(b c)^{m-1-i}-\sum_{i=0}^{m-1}(c b)^{i} \cdot r_{2} \cdot(b c)^{m-1-i} b=0, \\
b \cdot r_{1}+r_{1} \cdot b=0, \\
c \cdot r_{2}+r_{2} \cdot c=0 .
\end{gathered}
$$

Proposition 3.7. $\mathrm{HH}^{1}(R)=0$.

Proof. Assume that $\left(r_{1}, r_{2}\right) \in \operatorname{Ker} \delta^{1}$. Let

$$
r_{1}=\sum_{g \in G} \lambda_{g} g, \quad r_{2}=\sum_{g \in G} \mu_{g} g
$$

be canonical expansions (where $\lambda_{g}, \mu_{g} \in \mathbb{Z}$ ). The second equation in (3.7) implies

$$
\begin{aligned}
& \lambda_{(b c)^{i}}+\lambda_{(c b)^{i}}=0, \quad \lambda_{b(c b)^{i}}+\lambda_{(c b)^{i-1} c}=0 \text { for } 1 \leq i \leq m-1, \\
& \lambda_{1}=\lambda_{b}=\lambda_{(c b)^{m-1} c}=\lambda_{(b c)^{m}}=0 .
\end{aligned}
$$

By symmetry, the third equation in (3.7) yields

$$
\begin{aligned}
& \mu_{(b c)^{i}}+\mu_{(c b)^{i}}=0, \quad \mu_{c(b c)^{i}}+\mu_{(b c)^{i-1} b}=0 \text { for } 1 \leq i \leq m-1, \\
& \mu_{1}=\mu_{c}=\mu_{(b c)^{m-1} b}=\mu_{(b c)^{m}}=0 .
\end{aligned}
$$

Consequently, $r_{1}$ and $r_{2}$ are presented in the form

$$
\begin{aligned}
& r_{1}=\sum_{j=1}^{m-1} \lambda_{(b c)^{j}}\left((b c)^{j}-(c b)^{j}\right)+\sum_{j=1}^{m-1} \lambda_{b(c b)^{j}}\left(b(c b)^{j}-(c b)^{j-1} c\right), \\
& r_{2}=\sum_{j=1}^{m-1} \mu_{(b c)^{j}}\left((b c)^{j}-(c b)^{j}\right)+\sum_{j=1}^{m-1} \mu_{c(b c)^{j}}\left(c(b c)^{j}-(b c)^{j-1} b\right) .
\end{aligned}
$$


Since the elements $\left((b c)^{j}-(c b)^{j}, 0\right),\left(0,(b c)^{j}-(c b)^{j}\right), 1 \leq j \leq m-1$, lie in $\operatorname{Im} \delta^{0} \subset \operatorname{Ker} \delta^{1}$, subtracting a suitable linear combination of these elements from $\left(r_{1}, r_{2}\right)$, we may assume that in the initial elements $r_{1}, r_{2}$ we have

$$
\lambda_{(b c)^{j}}=0=\mu_{(b c)^{j}} \text { for } 1 \leq j \leq m-1 .
$$

Observe that

$$
\begin{aligned}
\sum_{i=0}^{m-1} c(b c)^{i} \cdot b(c b)^{j} \cdot(c b)^{m-1-i} & =\sum_{i=0}^{m-1}(c b)^{m+j}=m(b c)^{m-j} \\
\sum_{i=0}^{m-1} c(b c)^{i} \cdot(c b)^{j-1} c \cdot(c b)^{m-1-i} & =\sum_{i=0}^{m-1}(c b)^{i}(b c)^{j-1}(c b)^{m-1-i} \\
& =\sum_{i=0}^{m-1}(c b)^{m-j}=m(c b)^{m-j}
\end{aligned}
$$

for $1 \leq j \leq m-1$. Similarly,

$$
\begin{aligned}
& \sum_{i=0}^{m-1}(b c)^{i} \cdot b(c b)^{j} \cdot(c b)^{m-1-i} c=m(c b)^{m-j} \\
& \sum_{i=0}^{m-1}(b c)^{i} \cdot(c b)^{j-1} c \cdot(c b)^{m-1-i} c=m(b c)^{m-j} .
\end{aligned}
$$

Substituting (3.8) in the first equation in (3.7) and keeping relations (3.9) in mind, we obtain

$$
2 m \sum_{j=1}^{m-1}\left(\lambda_{b(c b)^{j}}+\mu_{c(b c)^{j}}\right)\left((c b)^{m-j}-(b c)^{m-j}\right)=0,
$$

whence $\lambda_{b(c b)^{j}}+\mu_{c(b c)^{j}}=0$ for all $1 \leq j \leq m-1$. This shows that $\operatorname{Ker} \delta^{1}$ is generated by the set (3.5), so that $\operatorname{Ker} \delta^{1}=\operatorname{Im} \delta^{0}$.

Proposition 3.8. The free $\mathbb{Z}$-module $\operatorname{Im} \delta^{1}$ admits a basis formed by the following elements:

$$
\begin{aligned}
& (0,2,0),(0,2 b, 0),(0,0,2),(0,0,2 c) ; \\
& \left(0,2 c(b c)^{m-1}, 0\right),\left(0,2(b c)^{m}, 0\right),\left(0,0,2 b(c b)^{m-1}\right),\left(0,0,2(b c)^{m}\right) ; \\
& \left(2 m\left((b c)^{j}-(c b)^{j}\right), 0,0\right), 1 \leq j \leq m-1 ; \\
& \left(m\left((c b)^{m-j}-(b c)^{m-j}\right),(b c)^{j}+(c b)^{j}, 0\right), 1 \leq j \leq m-1 ; \\
& \left(0, c(b c)^{j-1}+b(c b)^{j}, 0\right), 1 \leq j \leq m-1 ; \\
& \left(m\left((c b)^{m-j}-(b c)^{m-j}\right), 0,(b c)^{j}+(c b)^{j}\right), 1 \leq j \leq m-1 ; \\
& \left(0,0, b(c b)^{j-1}+c(b c)^{j}\right), 1 \leq j \leq m-1 .
\end{aligned}
$$

Proof. First, observe that for $0 \leq j \leq m-1$ we have

$$
\delta^{1}\left((b c)^{j}, 0\right)=\left(\Xi_{j}, c(b c)^{j-1}+(b c)^{j} b, 0\right),
$$

where

$$
\Xi_{j}:=-\sum_{i=0}^{m-1} c(c b)^{m-1-2 i-j}+\sum_{i=0}^{m-1}(c b)^{m-1-2 i-j} c .
$$


Then we obtain

$$
\begin{aligned}
c \cdot \Xi_{j} & =-\sum_{i=0}^{m-1}(c b)^{m-1-2 i-j}+\sum_{i=0}^{m-1}(b c)^{m-1-2 i-j} \\
& =-\sum_{i=0}^{m-1}(b c)^{-m+1+2 i+j}+\sum_{l=0}^{m-1}(b c)^{-m+1+2 l-j},
\end{aligned}
$$

where, in the second sum, we have changed the index of summation: $l:=m-1-i$. Consequently,

$$
\begin{aligned}
c \cdot \Xi_{j} & =-\sum_{i=0}^{m-1}(b c)^{m+1+2 i+j}+\sum_{l=0}^{m-1}(b c)^{m+1+2 l-j} \\
& =(b c)^{m+1-j}\left(-\sum_{i=0}^{m-1}(b c)^{2 i+2 j}+\sum_{l=0}^{m-1}(b c)^{2 l}\right)=0
\end{aligned}
$$

(the latter identity is obtained with the help of $(\underline{3.6})$ ), whence $\Xi_{j}=0$. Consequently, $\delta^{1}\left((b c)^{j}, 0\right)=\left(0, c(b c)^{j-1}+(b c)^{j} b, 0\right) \quad(1 \leq j \leq m-1)$ and $\delta^{1}(1,0)=(0,2 b, 0)$. By symmetry, $\delta^{1}\left(0,(c b)^{j}\right)=\left(0,0, b(c b)^{j-1}+(c b)^{j} c\right), 1 \leq j \leq m-1$, and $\delta^{1}(0,1)=$ $(0,0,2 c)$. Furthermore, it is easily seen that

$$
\begin{aligned}
& \delta^{1}(b, 0)=(0,2,0), \delta^{1}\left(c(b c)^{m-1}, 0\right)=\left(0,2(b c)^{m}, 0\right), \\
& \delta^{1}\left((b c)^{m}, 0\right)=\left(0,2 c(b c)^{m-1}, 0\right) ; \\
& \delta^{1}\left(b(c b)^{j}, 0\right)=\left(m\left((c b)^{m-j}-(b c)^{m-j}\right),(b c)^{j}+(c b)^{j}, 0\right), \quad 1 \leq j \leq m-1 ; \\
& \delta^{1}\left(b(c b)^{j}-c(b c)^{j-1}, 0\right)=\left(2 m\left((c b)^{m-j}-(b c)^{m-j}\right), 0,0\right), \quad 1 \leq j \leq m-1 .
\end{aligned}
$$

By symmetry, we also have

$$
\begin{aligned}
& \delta^{1}(0, c)=(0,0,2), \delta^{1}\left(0, b(c b)^{m-1}\right)=\left(0,0,2(b c)^{m}\right), \\
& \delta^{1}\left(0,(b c)^{m}\right)=\left(0,0,2 b(c b)^{m-1}\right) ; \\
& \delta^{1}\left(0, c(b c)^{j}\right)=\left(m\left((c b)^{m-j}-(b c)^{m-j}, 0,(b c)^{j}+(c b)^{j}, 0\right), \quad 1 \leq j \leq m-1 ;\right. \\
& \delta^{1}\left(0, c(b c)^{j}-b(c b)^{j-1}, 0\right)=\left(2 m\left((c b)^{m-j}-(b c)^{m-j}, 0,0\right), \quad 1 \leq j \leq m-1 .\right.
\end{aligned}
$$

It follows that the set of elements in (3.10) -3.16 generates $\operatorname{Im} \delta^{1}$. Since

$$
\operatorname{rk} \operatorname{Im} \delta^{1}=\operatorname{rk} R^{2}-\operatorname{rk} \operatorname{Ker} \delta^{1}=8 m-(3 m-3)=5 m+3
$$

and the cardinality of the set of generators indicated is also equal to $5 m+3$, this set is a basis of $\operatorname{Im} \delta^{1}$.

Since

$$
d_{2}^{Q}=\left(\begin{array}{cccc}
B^{+} & C^{+} & 0 & 0 \\
\rho^{-} & 0 & B^{-} & 0 \\
0 & -\omega^{-} & 0 & C^{-}
\end{array}\right)
$$


the condition $\left(r_{1}, r_{2}, r_{3}\right) \in \operatorname{Ker} \delta^{2}$ is equivalent to the following system of equations:

$$
\begin{aligned}
B^{+} * r_{1}+\rho^{-} * r_{2} & =0, \\
C^{+} * r_{1}-\omega^{-} * r_{3} & =0, \\
B^{-} * r_{2} & =0, \\
C^{-} * r_{3} & =0 .
\end{aligned}
$$

Proposition 3.9. The free $\mathbb{Z}$-module $\operatorname{Ker} \delta^{2}$ admits a basis formed by the elements (3.13) - (3.16) and by the following elements:

$$
\begin{aligned}
& (0,1,0),(0, b, 0),\left(0, c(b c)^{m-1}, 0\right),\left(0,(b c)^{m}, 0\right) ; \\
& (0,0,1),(0,0, c),\left(0,0, b(c b)^{m-1}\right),\left(0,0,(b c)^{m}\right) ; \\
& \left((b c)^{j}-(c b)^{j}, 0,0\right), \quad 1 \leq j \leq m-1 .
\end{aligned}
$$

The following fact, needed in the proof of this proposition, can be established by direct calculation.

Lemma 3.10. a) For any $r \in R$, we have $\rho^{-} * r=\left(B^{-} * r\right)(b c)^{m}$.

b) For any $r \in R$, we have $\omega^{-} * r=\left(C^{-} * r\right)(b c)^{m}$.

Proof of Proposition 3.9. Lemma 3.10 implies that system (3.18) is equivalent to

$$
\begin{aligned}
& B^{+} * r_{1}=0, \\
& C^{+} * r_{1}=0, \\
& B^{-} * r_{2}=0, \\
& C^{-} * r_{3}=0 .
\end{aligned}
$$

Assume that $x=\left(r_{1}, r_{2}, r_{3}\right) \in \operatorname{Ker} \delta^{2}$. Put $r_{2}=\sum_{g \in G} \mu_{g} g$ (with $\mu_{g} \in \mathbb{Z}$ ) and substitute this in the third equation in (3.22). It is easily seen that this equation is equivalent to the relations

$$
\mu_{(b c)^{i}}=\mu_{(c b)^{i}}, \quad \mu_{b(c b)^{i}}=\mu_{(c b)^{i-1} c} \text { for } 1 \leq i \leq m-1 .
$$

Consequently, the subgroup of $\operatorname{Ker} \delta^{2}$ that consists of elements of the form $\left(0, r_{2}, 0\right)$ with $r_{2} \in R$ is generated by the elements in (3.19), (3.14) and by the elements

$$
\left(0,(b c)^{i}+(c b)^{i}, 0\right) \text { for } 1 \leq i \leq m-1 .
$$

A similar analysis of the fourth equation in (3.22) shows that the subgroup of $\operatorname{Ker} \delta^{2}$ that consists of elements of the form $\left(0,0, r_{3}\right)$ with $r_{3} \in R$ is generated by the elements in (3.20), (3.16) and the elements

$$
\left(0,0,(b c)^{i}+(c b)^{i}\right) \text { for } 1 \leq i \leq m-1 .
$$

Substituting the canonical expansion $r_{1}=\sum_{g \in G} \lambda_{g} g\left(\lambda_{g} \in \mathbb{Z}\right)$ in the first equation in (3.22), we obtain

$$
\begin{aligned}
& \lambda_{1}=\lambda_{b}=\lambda_{c(b c)^{m-1}}=\lambda_{(b c)^{m}}=0 \\
& \lambda_{(b c)^{i}}+\lambda_{(c b)^{i}}=0 \text { for } 1 \leq i \leq m-1 \\
& \lambda_{b(c b)^{i}}+\lambda_{(c b)^{i-1} c}=0 \text { for } 1 \leq i \leq m-1 .
\end{aligned}
$$

Similarly, the second equation in (3.22) implies that

$$
\begin{aligned}
& \lambda_{c}=\lambda_{b(c b)^{m-1}}=0 \\
& \lambda_{c(b c)^{i}}+\lambda_{(b c)^{i-1} b}=0 \text { for } 1 \leq i \leq m-1 .
\end{aligned}
$$


Then (using the relation $\lambda_{b}=\lambda_{c}=0$ ), from (3.27) and (3.28) we deduce that $\lambda_{b(c b)^{i}}=$ $\lambda_{c(b c)^{i}}=0$ for $0 \leq i \leq m-1$. Hence, the subgroup of $\operatorname{Ker} \delta^{2}$ that consists of elements of the form $\left(r_{1}, 0,0\right)$ with $r_{1} \in R$ is generated by the elements in (3.21). Consequently, $\operatorname{Ker} \delta^{2}$ is generated by the set of elements in (3.19), (3.20), (3.21), (3.14), (3.16) and the elements in (3.23) and (3.24). But it is clear that the set of elements in (3.23) and (3.24) can be replaced by (3.13) and (3.15), respectively. It remains to observe that the cardinality of the resulting set of generators of the $\mathbb{Z}$-module $\operatorname{Ker} \delta^{2}$ is equal to $5 m+3=\operatorname{rk} \operatorname{Im} \delta^{1}$ (see (3.17) $)$; hence, this set is linearly independent (over $\mathbb{Z}$ ).

Proposition 3.11. The group $\mathrm{HH}^{2}(R)$ is generated by the set of cohomology classes of the following elements:

$$
\begin{aligned}
& u_{0}^{(i)}:=\left((b c)^{i}-(c b)^{i}, 0,0\right) \text { for } 1 \leq i \leq m-1 ; \\
& u_{1}:=(0,1,0), \quad u_{1}^{\prime}:=(0,0,1), \quad u_{2}:=(0, b, 0), \quad u_{2}^{\prime}:=(0,0, c), \\
& u_{3}:=\left(0, c(b c)^{m-1}, 0\right), \quad u_{3}^{\prime}:=\left(0,0, b(c b)^{m-1}\right), \\
& u_{4}:=\left(0,(b c)^{m}, 0\right), \quad u_{4}^{\prime}:=\left(0,0,(b c)^{m}\right),
\end{aligned}
$$

which satisfy the relations

$$
\begin{aligned}
& 2 m u_{0}^{(i)}=0 \text { for } 1 \leq i \leq m-1 \\
& 2 u_{j}=2 u_{j}^{\prime}=0 \text { for } j \in\{1,2,3,4\} .
\end{aligned}
$$

In particular, we have

$$
\mathrm{HH}^{2}(R) \simeq \mathbb{Z}_{2 m}^{m-1} \oplus \mathbb{Z}_{2}^{8} .
$$

Proof. This follows from the fact that the bases of the groups $\operatorname{Ker} \delta^{2}$ and $\operatorname{Im} \delta^{1}$ presented in Propositions 3.9 and 3.8 (respectively) are the so-called stacked bases.

Remark 3.12. In the sequel, if $f \in \operatorname{Ker} \delta^{n}$ is an $n$-cocycle, its cohomology class cl $f \in$ $\mathrm{HH}^{n}(R)$ is often still denoted by $f$.

Proposition 3.13. The free $\mathbb{Z}$-module $\operatorname{Im} \delta^{2}$ admits a basis formed by the following elements:

$$
\begin{aligned}
& (2 b, 2 c, 0,0), \quad\left(2 c(b c)^{m-1}, 2 b(c b)^{m-1}, 0,0\right) \\
& \left((b c)^{i} b+c(b c)^{i-1},(c b)^{i} c+b(c b)^{i-1}, 0,0\right), \quad 1 \leq i \leq m-1 ; \\
& \left((b c)^{i}+(c b)^{i},(b c)^{i+1}+(c b)^{i+1}, 0,0\right), \quad 0 \leq i \leq m-1 ; \\
& \left((b c)^{i+1}+(c b)^{i+1},(b c)^{i}+(c b)^{i}, 0,0\right), \quad 0 \leq i \leq m-1 ; \\
& \left(b(c b)^{m-i}-(c b)^{m-1-i} c, 0, c(b c)^{i-1}-(b c)^{i} b, 0\right), \quad 1 \leq i \leq m-1 ; \\
& \left((b c)^{m-i}-(c b)^{m-i}, 0,(c b)^{i}-(b c)^{i}, 0\right), \quad 1 \leq i \leq m-1 ; \\
& \left(0, c(b c)^{m-i}-(b c)^{m-1-i} b, 0, c(b c)^{i}-(b c)^{i-1} b\right), \quad 1 \leq i \leq m-1 ; \\
& \left(0,(b c)^{m-i}-(c b)^{m-i}, 0,(b c)^{i}-(c b)^{i}\right), \quad 1 \leq i \leq m-1 .
\end{aligned}
$$


Proof. It is verified directly that the elements on the list (3.29)-(3.36) coincide with the following values of the differential $\delta^{2}$ :

$$
\begin{array}{llll}
\delta^{2}(1,0,0), & \delta^{2}\left((b c)^{m}, 0,0\right), \delta^{2}\left((b c)^{i}, 0,0\right) & (1 \leq i \leq m-1), \\
\delta^{2}\left(b(c b)^{i}, 0,0\right) & (0 \leq i \leq m-1), & \delta^{2}\left(c(b c)^{i}, 0,0\right) & (0 \leq i \leq m-1), \\
\delta^{2}\left(0,(b c)^{i}, 0\right) & (1 \leq i \leq m-1), & \delta^{2}\left(0, b(c b)^{i}, 0\right) & (1 \leq i \leq m-1), \\
\delta^{2}\left(0,0,(b c)^{i}\right) & (1 \leq i \leq m-1), & \delta^{2}\left(0,0, c(b c)^{i}\right) & (1 \leq i \leq m-1) .
\end{array}
$$

Also, it is easily seen that this set of elements generates $\operatorname{Im} \delta^{2}$ and has cardinality equal to $7 m-3$. Since $\operatorname{rk} \operatorname{Im} \delta^{2}=\operatorname{rk} R^{3}-\operatorname{rk} \operatorname{Ker} \delta^{2}=12 m-(5 m+3)=7 m-3$, this set is linearly independent.

Since

$$
d_{3}^{Q}=\left(\begin{array}{ccccc}
\zeta^{+} & B^{-} & 0 & 0 & 0 \\
-\eta^{+} & 0 & C^{-} & 0 & 0 \\
0 & -\rho^{-} & 0 & B^{+} & 0 \\
0 & 0 & \omega^{-} & 0 & C^{+}
\end{array}\right)
$$

the condition $\left(r_{1}, r_{2}, r_{3}, r_{4}\right) \in \operatorname{Ker} \delta^{3}$ is equivalent to the following system of equations:

$$
\begin{aligned}
\zeta^{+} * r_{1}-\eta^{+} * r_{2} & =0, \\
B^{-} * r_{1}-\rho^{-} * r_{3} & =0, \\
C^{-} * r_{2}+\omega^{-} * r_{4} & =0, \\
B^{+} * r_{3} & =0, \\
C^{+} * r_{4} & =0 .
\end{aligned}
$$

Proposition 3.14. a) If $m$ is even, then the free $\mathbb{Z}$-module $\operatorname{Ker} \delta^{3}$ admits a basis formed by the elements in (3.33)-(3.36) and by the following elements:

$$
\begin{aligned}
& (b, c, 0,0),\left(c(b c)^{m-1}, b(c b)^{m-1}, 0,0\right) ; \\
& \left(b(c b)^{i}+c(b c)^{i-1}, c(b c)^{i}+b(c b)^{i-1}, 0,0\right), \quad 1 \leq i \leq m-1 ; \\
& \left(1-(b c)^{m}, 0,0,0\right),\left(0,1-(b c)^{m}, 0,0\right) ; \\
& \left((b c)^{i}+(c b)^{i},(b c)^{i+1}+(c b)^{i+1}, 0,0\right), \quad 0 \leq i \leq m-2 ; \\
& \left((b c)^{i+1}+(c b)^{i+1},(b c)^{i}+(c b)^{i}, 0,0\right), \quad 0 \leq i \leq m-2 .
\end{aligned}
$$

b) If $m$ is odd, then in order to get a basis of the $\mathbb{Z}$-module $\operatorname{Ker} \delta^{3}$, it suffices to replace the elements (3.40) in the set described in part a) by the elements

$$
\left(1,(b c)^{m}, 0,0\right),\left((b c)^{m}, 1,0,0\right) .
$$

Lemma 3.15. a) For the homomorphisms (of Abelian groups)

$$
\begin{aligned}
& f_{b}^{+}: R \rightarrow R, \quad f_{b}^{+}(r)=B^{+} * r, \\
& f_{b}^{-}: R \rightarrow R, \quad f_{b}^{-}(r)=B^{-} * r,
\end{aligned}
$$

we have $\operatorname{Ker} f_{b}^{+}=\operatorname{Im} f_{b}^{-}$.

b) For the homomorphisms

$$
\begin{aligned}
& f_{c}^{+}: R \rightarrow R, \quad f_{c}^{+}(r)=C^{+} * r, \\
& f_{c}^{-}: R \rightarrow R, \quad f_{c}^{-}(r)=C^{-} * r,
\end{aligned}
$$

we have $\operatorname{Ker} f_{c}^{+}=\operatorname{Im} f_{c}^{-}$. 
Proof. a) The inclusion $\operatorname{Im} f_{b}^{-} \subset \operatorname{Ker} f_{b}^{+}$follows from the fact that $f_{b}^{+}$and $f_{b}^{-}$are induced by the $\Lambda$-homomorphisms $\left(B^{+}\right)^{*},\left(B^{-}\right)^{*}: \Lambda \rightarrow \Lambda$, for which we have $\left(B^{-}\right)^{*} \circ\left(B^{+}\right)^{*}=0$. Next, if $f_{b}^{+}(r)=0$ with $r=\sum_{g \in G} \lambda_{g} g \in R$, then relations (3.25)-3.27) are fulfilled, whence we have

$$
\begin{aligned}
r & =\sum_{i=1}^{m-1} \lambda_{(b c)^{i}}\left((b c)^{i}-(c b)^{i}\right)+\sum_{i=1}^{m-1} \lambda_{b(c b)^{i}}\left(b(c b)^{i}-c(b c)^{i-1}\right) \\
& =f_{b}^{-}\left(-\sum_{i=1}^{m-1} \lambda_{(b c)^{i}} b(c b)^{i}+\sum_{i=1}^{m-1} \lambda_{b(c b)^{i}}(c b)^{i}\right)
\end{aligned}
$$

Part b) of the lemma is proved similarly.

Proof of Proposition 3.14. Assume that $x=\left(r_{1}, r_{2}, r_{3}, r_{4}\right) \in \operatorname{Ker} \delta^{3}$. Using Lemma 3.15, from the fourth and fifth equations in (3.37) we deduce the relations $r_{3}=B^{-} * u$, $r_{3}=C^{-} * v$ for some $u, v \in R$. The proof of Proposition 3.13 shows that the element $\delta^{2}(0, u, v)=\left(\rho^{-} * u,-\omega^{-} * v, r_{3}, r_{4}\right)$ can be represented as a linear combination of elements in (3.33) -(3.36). Replacing $x$ by $x-\delta^{2}(0, u, v)=\left(r_{1}-\rho^{-} * u, r_{2}+\omega^{-} * v, 0,0\right)$, we may assume that in the initial element $x \in \operatorname{Ker} \delta^{3}$ we have $r_{3}=r_{4}=0$.

We consider the canonical expansions

$$
r_{1}=\sum_{g \in G} \lambda_{g} g, \quad r_{2}=\sum_{g \in G} \mu_{g} g \quad\left(\lambda_{g}, \mu_{g} \in \mathbb{Z}\right)
$$

and substitute formulas (3.44) in the first equation in (3.37). It is easily verified that $\zeta^{+} * r, \eta^{+} * r \in Z(R)$ for any $r \in R$. Hence, the element on the left-hand side of this equation can be expanded in a basis of $Z(R)$ (see Proposition 3.1).

We introduce an auxiliary notation:

$$
\Phi_{1}:=(b c)^{m} \theta_{b}, \quad \Phi_{2}:=(b c)^{m} \theta_{c} .
$$

By Corollary 3.5. if $m$ is even, then $\Phi_{1}=\theta_{b}$ and $\Phi_{2}=\theta_{c}$, and if $m$ is odd, then $\Phi_{1}=\theta_{c}$ and $\Phi_{2}=\theta_{b}$. A direct calculation shows that

$$
\begin{aligned}
& \zeta^{+} * r_{1}-\eta^{+} * r_{2}=2 m\left(\lambda_{c(b c)^{m-1}}-\mu_{b(c b)^{m-1}}\right) \cdot 1+2 m\left(\lambda_{b}-\mu_{c}\right) \cdot(b c)^{m} \\
& +2\left(\sum_{j \text { is even }} \lambda_{(b c)^{j}}+\sum_{j \text { is even }} \lambda_{(c b)^{j}}-\sum_{j \text { is odd }} \mu_{(b c)^{j}}-\sum_{j \text { is odd }} \mu_{(c b)^{j}}\right) \cdot \Phi_{1} \\
& +2\left(\sum_{j \text { is odd }} \lambda_{(b c)^{j}}+\sum_{j \text { is odd }} \lambda_{(c b)^{j}}-\sum_{j \text { is even }} \mu_{(b c)^{j}}-\sum_{j \text { is even }} \mu_{(c b)^{j}}\right) \cdot \Phi_{2} \\
& +m \sum_{j=1}^{m-1}\left(\lambda_{b(c b)^{m-j}}+\lambda_{c(b c)^{m-1-j}}-\mu_{b(c b)^{m-1-j}}-\mu_{c(b c)^{m-j}}\right) \cdot\left((b c)^{j}+(c b)^{j}\right) .
\end{aligned}
$$

Consequently, the first equation in (3.37) is equivalent to the system of equations (over $K)$ for $\lambda_{g}, \mu_{g}(g \in G)$ that is obtained by equating the coefficients of the decomposition (3.46) to zero.

Now, the second and third equations in (3.37) imply the following relations (cf. the proof of Proposition [3.9):

$$
\begin{aligned}
& \lambda_{(b c)^{j}}=\lambda_{(c b)^{j}}, \lambda_{b(c b)^{j}}=\lambda_{c(b c)^{j-1}} \text { for } 1 \leq j \leq m-1 ; \\
& \mu_{(b c)^{j}}=\mu_{(c b)^{j}}, \mu_{c(b c)^{j}}=\mu_{b(c b)^{j-1}} \text { for } 1 \leq j \leq m-1 .
\end{aligned}
$$

A direct verification shows that the elements in (3.38) and (3.39) lie in $\operatorname{Ker} \delta^{3}$. Consequently, subtracting suitable multiples of these elements, we may assume that for the 
initial element $x \in \operatorname{Ker} \delta^{3}$ we have

$$
\begin{aligned}
& \lambda_{b}=\lambda_{c(b c)^{m-1}}=\mu_{c}=\mu_{b(c b)^{m-1}}=0 ; \\
& \lambda_{b(c b)^{j}}=\lambda_{c(b c)^{j-1}}=\mu_{c(b c)^{j}}=\mu_{b(c b)^{j-1}}=0 \text { for } 1 \leq j \leq m-1 .
\end{aligned}
$$

Assume additionally that $m$ is even: $m=2 k$. In this case,

$$
\left(1-(b c)^{m}, 0,0,0\right),\left(0,1-(b c)^{m}, 0,0\right) \in \operatorname{Ker} \delta^{3} .
$$

Adding to $x$ suitable multiples of these elements, we may assume additionally that $\lambda_{(b c)^{m}}=\mu_{(b c)^{m}}=0$. Then the first equation in (3.37), combined with (3.47) and (3.48), implies the following relations for the remaining coefficients of the canonical expansions (3.44):

$$
\begin{aligned}
\lambda_{1}+2 \sum_{t=1}^{k-1} \lambda_{(b c)^{2 t}} & =2 \sum_{t=1}^{k} \mu_{(b c)^{2 t-1}} \\
\mu_{1}+2 \sum_{t=1}^{k-1} \mu_{(b c)^{2 t}} & =2 \sum_{t=1}^{k} \lambda_{(b c)^{2 t-1}} .
\end{aligned}
$$

It is easily seen that the set of (integral) solutions of equation (3.49) is generated by the elements

$$
\begin{array}{ll}
\left((b c)^{2 i}+(c b)^{2 i},(b c)^{2 i+1}+(c b)^{2 i+1}, 0,0\right), & 0 \leq i \leq \frac{m-2}{2} \\
\left((b c)^{2 i}+(c b)^{2 i},(b c)^{2 i-1}+(c b)^{2 i-1}, 0,0\right), & 1 \leq i \leq \frac{m-2}{2} .
\end{array}
$$

By symmetry, the set of solutions of equation (3.50) is generated by the elements

$$
\begin{array}{ll}
\left((b c)^{2 i+1}+(c b)^{2 i+1},(b c)^{2 i}+(c b)^{2 i}, 0,0\right), & 0 \leq i \leq \frac{m-2}{2} \\
\left((b c)^{2 i-1}+(c b)^{2 i-1},(b c)^{2 i}+(c b)^{2 i}, 0,0\right), & 1 \leq i \leq \frac{m-2}{2}
\end{array}
$$

Clearly, the union of the sets of elements in (3.51)-(3.54) coincides with the union of the sets (3.41) and (3.42). Consequently, we have proved that the set of elements described in Proposition 3.14a) generates Ker $\delta^{3}$. Since its cardinality is equal to $\operatorname{rk} \operatorname{Im} \delta^{2}=7 m-3$, this set is linearly independent.

Now assume that $m$ is odd. Then

$$
\left(1,(b c)^{m}, 0,0\right),\left((b c)^{m}, 1,0,0\right) \in \operatorname{Ker} \delta^{3} .
$$

Adding to $x$ suitable multiples of these elements, again we may assume that $\lambda_{(b c)^{m}}=$ $\mu_{(b c)^{m}}=0$. Then we can argue much as in the preceding case to obtain the desired statement.

Proposition 3.16. a) If $m$ is even, then the group $\mathrm{HH}^{3}(R)$ is generated by the set of cohomology classes of the following elements:

$$
\begin{array}{ll}
v_{1}:=(b, c, 0,0), & v_{1}^{\prime}:=\left(c(b c)^{m-1}, b(c b)^{m-1}, 0,0\right), \\
v_{2}:=\left(1-(b c)^{m}, 0,0,0\right), & v_{2}^{\prime}:=\left(0,1-(b c)^{m}, 0,0\right),
\end{array}
$$

which satisfy the relations

$$
2 v_{j}=2 v_{j}^{\prime}=0 \text { for } j \in\{1,2\} \text {. }
$$


b) If $m$ is odd, then the group $\mathrm{HH}^{3}(R)$ is generated by the set of cohomology classes of the elements

$$
\widetilde{v}_{2}:=\left(1,(b c)^{m}, 0,0\right), \quad \widetilde{v}_{2}^{\prime}:=\left((b c)^{m}, 1,0,0\right),
$$

together with the cohomology classes of the elements $v_{1}, v_{1}^{\prime}$ in (3.55), which satisfy the relations

$$
2 v_{1}=2 v_{1}^{\prime}=2 \widetilde{v}_{2}=2 \widetilde{v}_{2}^{\prime}=0
$$

In particular, for any $m \geq 2$ we have

$$
\mathrm{HH}^{3}(R) \simeq \mathbb{Z}_{2}^{4} .
$$

Proof. a) Assume that $m$ is even: $m=2 k$. Since

$$
\begin{aligned}
2\left(1-(b c)^{m}, 0,0,0\right)= & \sum_{t=0}^{k-1}\left((b c)^{2 t}+(c b)^{2 t},(b c)^{2 t+1}+(c b)^{2 t+1}, 0,0\right) \\
& -\sum_{t=1}^{k}\left((b c)^{2 t}+(c b)^{2 t},(b c)^{2 t-1}+(c b)^{2 t-1}, 0,0\right),
\end{aligned}
$$

we can replace the element $\left(2(b c)^{m},(b c)^{m-1}+(c b)^{m-1}, 0,0\right)$ in the basis of the $\mathbb{Z}$-module $\operatorname{Im} \delta^{2}$ indicated in Proposition 3.13 (see (3.32) $)$ by the element $2\left(1-(b c)^{m}, 0,0,0\right)$. By symmetry, we can replace the element $\left((b c)^{m-1}+(c b)^{m-1}, 2(b c)^{m}, 0,0\right)$ in this basis by the element $2\left(0,1-(b c)^{m}, 0,0\right)$. The desired statement follows from the fact that the new basis for $\operatorname{Im} \delta^{2}$ and the basis of $\operatorname{Ker} \delta^{3}$ described in Proposition 3.14 are stacked bases.

b) Assume that $m$ is odd: $m=2 k+1$. Then

$$
\begin{aligned}
2\left(1,(b c)^{m}, 0,0\right)= & \sum_{t=0}^{k}\left((b c)^{2 t}+(c b)^{2 t},(b c)^{2 t+1}+(c b)^{2 t+1}, 0,0\right) \\
& -\sum_{t=1}^{k}\left((b c)^{2 t}+(c b)^{2 t},(b c)^{2 t-1}+(c b)^{2 t-1}, 0,0\right) .
\end{aligned}
$$

Hence, we can replace the element $\left((b c)^{m-1}+(c b)^{m-1}, 2(b c)^{m},(0,0)\right.$ in the basis of $\operatorname{Im} \delta^{2}$ by the element $2\left(1,(b c)^{m}, 0,0\right)$. By symmetry, the element $\left(2(b c)^{m},(b c)^{m-1}+\right.$ $\left.(c b)^{m-1}, 0,0\right)$ in the basis of $\operatorname{Im} \delta^{2}$ can be replaced by $2\left((b c)^{m}, 1,0,0\right)$. As in the preceding case, we obtain stacked bases of the modules $\operatorname{Im} \delta^{2} \subset \operatorname{Ker} \delta^{3}$, and the desired statement follows.

Proposition 3.17. The free $\mathbb{Z}$-module $\operatorname{Im} \delta^{3}$ admits a basis formed by the following elements:

$$
\begin{aligned}
& \left(2 \Phi_{1}, \mathrm{O}_{4}\right), \quad\left(2 m(b c)^{m}, \mathrm{O}_{4}\right), \quad\left(2 m, \mathrm{O}_{4}\right) ; \\
& \left(m\left((b c)^{m-j}+(c b)^{m-j}\right),(c b)^{j}-(b c)^{j}, \mathrm{O}_{3}\right), \quad 1 \leq j \leq m-1 ; \\
& \left(2 \Phi_{1}(b c)^{j}, c(b c)^{j-1}-b(c b)^{j}, \mathrm{O}_{3}\right), \quad 1 \leq j \leq m-1 ; \\
& \left(2 m\left((b c)^{m-j}+(c b)^{m-j}\right), \mathrm{O}_{4}\right), \quad 1 \leq j \leq m-1 ; \\
& \left(2 \Phi_{2}, \mathrm{O}_{4}\right), \quad\left(\mathrm{O}_{3}, 2 b, 0\right), \quad\left(\mathrm{O}_{3}, 2,0\right)
\end{aligned}
$$




$$
\begin{aligned}
& \left(m\left((b c)^{m-j}+(c b)^{m-j}\right), 0,(b c)^{j}-(c b)^{j}, \mathrm{O}_{2}\right), \quad 1 \leq j \leq m-1 ; \\
& \left(2 \Phi_{2}(b c)^{j}, 0,(b c)^{j-1} b-c(b c)^{j}, \mathrm{O}_{2}\right), \quad 1 \leq j \leq m-1 ; \\
& \left(0,(c b)^{m-j}-(b c)^{m-j}, 0,(b c)^{j}+(c b)^{j}, 0\right), \quad 1 \leq j \leq m-1 ; \\
& \left(0, c(b c)^{m-j-1}-b(c b)^{m-j}, 0, c(b c)^{j-1}+b(c b)^{j}, 0\right), \quad 1 \leq j \leq m-1 ; \\
& \left(\mathrm{O}_{3}, 2(b c)^{m}, 0\right), \quad\left(\mathrm{O}_{3}, 2 c(b c)^{m-1}, 0\right) ; \\
& \left(\mathrm{O}_{4}, 2 c\right), \quad\left(\mathrm{O}_{4}, 2\right), \quad\left(\mathrm{O}_{4}, 2(b c)^{m}\right), \quad\left(\mathrm{O}_{4}, 2 b(c b)^{m-1}\right) ; \\
& \left(\mathrm{O}_{2},(c b)^{m-j}-(b c)^{m-j}, 0,(b c)^{j}+(c b)^{j}\right), \quad 1 \leq j \leq m-1 ; \\
& \left(\mathrm{O}_{2}, c(b c)^{m-j}-b(c b)^{m-j-1}, 0, c(b c)^{j}+b(c b)^{j-1}\right), \quad 1 \leq j \leq m-1 .
\end{aligned}
$$

Remark 3.18. Recall that $\Phi_{1}$ and $\Phi_{2}$ were defined in (3.45).

Proof of Proposition 3.17. It is verified directly that the elements on the list (3.56)-(3.68) coincide with the following values of the differential $\delta^{3}$ :

$$
\begin{aligned}
& \delta^{3}\left(1, \mathrm{O}_{3}\right), \delta^{3}\left(b, \mathrm{O}_{3}\right), \quad \delta^{3}\left(c(b c)^{m-1}, \mathrm{O}_{3}\right) ; \\
& \delta^{3}\left(b(c b)^{j}, \mathrm{O}_{3}\right) \quad(1 \leq j \leq m-1) ; \delta^{3}\left((b c)^{j}, \mathrm{O}_{3}\right) \quad(1 \leq j \leq m-1) ; \\
& \delta^{3}\left(b(c b)^{j}+c(b c)^{j-1}, \mathrm{O}_{3}\right) \quad(1 \leq i \leq m-1) ; \\
& \delta^{3}\left(0,-1, \mathrm{O}_{2}\right), \delta^{3}\left(\mathrm{O}_{2}, 1,0\right), \delta^{3}\left(\mathrm{O}_{2}, b, 0\right) ; \\
& \delta^{3}\left(0,-b(c b)^{j-1}, \mathrm{O}_{2}\right) \quad(1 \leq j \leq m-1) ; \delta^{3}\left(0,-(b c)^{j}, \mathrm{O}_{2}\right) \quad(1 \leq j \leq m-1) ; \\
& \delta^{3}\left(\mathrm{O}_{2}, b(c b)^{j}, 0\right) \quad(1 \leq j \leq m-1) ; \delta^{3}\left(\mathrm{O}_{2},(b c)^{j}, 0\right) \quad(1 \leq j \leq m-1) ; \\
& \delta^{3}\left(\mathrm{O}_{2}, c(b c)^{m-1}, 0\right), \delta^{3}\left(\mathrm{O}_{2},(b c)^{m}, 0\right), \delta^{3}\left(\mathrm{O}_{3}, 1\right), \delta^{3}\left(\mathrm{O}_{3}, c\right) ; \\
& \delta^{3}\left(\mathrm{O}_{3}, b(c b)^{m-1}\right), \delta^{3}\left(\mathrm{O}_{3},(b c)^{m}\right) ; \\
& \delta^{3}\left(\mathrm{O}_{3}, c(b c)^{j}, 0\right) \quad(1 \leq j \leq m-1) ; \quad \delta^{3}\left(\mathrm{O}_{3},(b c)^{j}\right) \quad(1 \leq j \leq m-1) .
\end{aligned}
$$

Using Proposition 3.14, we easily see that these elements generate $\operatorname{Im} \delta^{3}$; moreover, the cardinality of the set of elements in (3.56) $-(3.68)$ is equal to $9 m+3$. Since $\operatorname{rk} \operatorname{Im} \delta^{3}=$ $\operatorname{rk} R^{4}-\operatorname{rk} \operatorname{Ker} \delta^{2}=16 m-(7 m-3)=9 m+3$, this set is linearly independent.

Remark 3.19. For what follows, we describe another basis of $\operatorname{Im} \delta^{3}$; namely, if in the basis presented in Proposition 3.17 we replace the subset of elements of the form (3.58), (3.62), (3.64), and (3.68) by the family

$$
\begin{aligned}
& \left(0, c(b c)^{j-1}-b(c b)^{j}, \mathrm{O}_{3}\right), \quad\left(\mathrm{O}_{2},(b c)^{j-1} b-c(b c)^{j}, \mathrm{O}_{2}\right), \\
& \left(\mathrm{O}_{3}, c(b c)^{j-1}+b(c b)^{j}, 0\right), \quad\left(\mathrm{O}_{4}, c(b c)^{j}+b(c b)^{j-1}\right)
\end{aligned}
$$

for all $1 \leq j \leq m-1$, then we obtain a basis of $\operatorname{Im} \delta^{3}$.

Proposition 3.20. The free $\mathbb{Z}$-module Ker $\delta^{4}$ admits a basis formed by the following elements:

$$
\begin{aligned}
& \left(1, \mathrm{O}_{4}\right),\left(\theta_{b}, \mathrm{O}_{4}\right),\left(\theta_{c}, \mathrm{O}_{4}\right),\left((b c)^{m}, \mathrm{O}_{4}\right) ; \\
& \left((b c)^{i}+(c b)^{i}, \mathrm{O}_{4}\right), 1 \leq i \leq m-1 ; \\
& \left(0,(b c)^{i}-(c b)^{i}, \mathrm{O}_{3}\right), 1 \leq i \leq m-1 ;
\end{aligned}
$$




$$
\begin{aligned}
& \left(0, b(c b)^{i}-c(b c)^{i-1}, \mathrm{O}_{3}\right), 1 \leq i \leq m-1 ; \\
& \left(\mathrm{O}_{2},(b c)^{i}-(c b)^{i}, \mathrm{O}_{2}\right), 1 \leq i \leq m-1 ; \\
& \left(\mathrm{O}_{2}, c(b c)^{i}-b(c b)^{i-1} b, \mathrm{O}_{2}\right), 1 \leq i \leq m-1 ; \\
& \left(\mathrm{O}_{3}, 1,0\right),\left(\mathrm{O}_{3}, b, 0\right),\left(\mathrm{O}_{3}, c(b c)^{m-1}, 0\right),\left(\mathrm{O}_{3},(b c)^{m}, 0\right) ; \\
& \left(\mathrm{O}_{3},(b c)^{i}+(c b)^{i}, 0\right), 1 \leq i \leq m-1 ; \\
& \left(\mathrm{O}_{3}, b(c b)^{i}+c(b c)^{i-1}, 0\right), 1 \leq i \leq m-1 ; \\
& \left(\mathrm{O}_{4}, 1\right),\left(\mathrm{O}_{4}, c\right),\left(\mathrm{O}_{4}, b(c b)^{m-1}\right),\left(\mathrm{O}_{4},(b c)^{m}\right) ; \\
& \left(\mathrm{O}_{4},(b c)^{i}+(c b)^{i}\right), 1 \leq i \leq m-1 ; \\
& \left(\mathrm{O}_{4}, c(b c)^{i}+b(c b)^{i-1}\right), 1 \leq i \leq m-1 .
\end{aligned}
$$

Proof. Since

$$
d_{4}^{Q}=\left(\begin{array}{cccccc}
B^{-} & C^{-} & 0 & 0 & 0 & 0 \\
-\rho^{+} & 0 & B^{+} & 0 & 0 & 0 \\
0 & \omega^{+} & 0 & C^{+} & 0 & 0 \\
0 & 0 & \rho^{-} & 0 & B^{-} & 0 \\
0 & 0 & 0 & -\omega^{-} & 0 & C^{-}
\end{array}\right),
$$

the condition $\left(r_{1}, r_{2}, r_{3}, r_{4}, r_{5}\right) \in \operatorname{Ker} \delta^{4}$ is equivalent to the folowing system of equations:

$$
\begin{gathered}
B^{-} * r_{1}-\rho^{+} * r_{2}=0, \\
C^{-} * r_{1}+\omega^{+} * r_{3}=0, \\
B^{+} * r_{2}+\rho^{-} * r_{4}=0, \\
C^{+} * r_{3}-\omega^{-} * r_{5}=0, \\
B^{-} * r_{4}=0, \\
C^{-} * r_{5}=0 .
\end{gathered}
$$

Using Lemma 3.10, we observe that this system is equivalent to the following relations:

$$
\begin{array}{lll}
B^{-} * r_{1}=0 ; & B^{+} * r_{2}=0 ; & B^{-} * r_{4}=0 ; \\
C^{-} * r_{1}=0 ; & C^{+} * r_{3}=0 ; & C^{-} * r_{5}=0
\end{array}
$$

(to see this, we simplify system (3.73) "from bottom to top"). Next, using Proposition 3.1 and arguments in the proof of Proposition 3.9, we arrive at the above basis of $\operatorname{Ker} \delta^{4}$.

Proposition 3.21. The group $\mathrm{HH}^{4}(R)$ is generated by the set of cohomology classes of the following elements:

$$
\begin{aligned}
& w_{0}^{(i)}:=\left((b c)^{i}+(c b)^{i}, \mathrm{O}_{4}\right) \text { for } 1 \leq i \leq m-1 ; \\
& w_{1}:=\left(1, \mathrm{O}_{4}\right), w_{1}^{\prime}:=\left((b c)^{m}, \mathrm{O}_{4}\right), w_{2}:=\left(\theta_{b}, \mathrm{O}_{4}\right), w_{2}^{\prime}:=\left(\theta_{c}, \mathrm{O}_{4}\right), \\
& w_{3}:=\left(\mathrm{O}_{3}, 1,0\right), w_{3}^{\prime}:=\left(\mathrm{O}_{4}, 1\right), w_{4}:=\left(\mathrm{O}_{3}, b, 0\right), w_{4}^{\prime}:=\left(\mathrm{O}_{4}, c\right), \\
& w_{5}:=\left(\mathrm{O}_{3}, c(b c)^{m-1}, 0\right), w_{5}^{\prime}:=\left(\mathrm{O}_{4}, b(c b)^{m-1}\right), \\
& w_{6}:=\left(\mathrm{O}_{3},(b c)^{m}, 0\right), w_{6}^{\prime}:=\left(\mathrm{O}_{4},(b c)^{m}\right),
\end{aligned}
$$


which satisfy the relations

$$
\begin{aligned}
& 2 m w_{0}^{(i)}=0 \text { for } 1 \leq i \leq m-1 ; 2 m w_{1}=2 m w_{1}^{\prime}=0 \\
& 2 w_{j}=2 w_{j}^{\prime}=0 \text { for } 2 \leq j \leq 6 .
\end{aligned}
$$

In particular, we have

$$
\mathrm{HH}^{4}(R) \simeq \mathbb{Z}_{2 m}^{m+1} \oplus \mathbb{Z}_{2}^{10}
$$

Proof. In the basis of the group Ker $\delta^{4}$ described in Proposition 3.20, we replace the subset of elements of the form (3.69), (3.70), (3.71), (3.72) by the set of elements of the form (3.57), (3.61), (3.63), and (3.67). It is clear that the resulting collection is a basis for $\operatorname{Ker} \delta^{4}$. Moreover, this new basis and the basis of the group $\operatorname{Im} \delta^{3}$ presented in Remark 3.19 are stacked bases, whence the desired statement follows.

Proposition 3.22. For any $n \geq 5$, we have

$$
\mathrm{HH}^{n}(R) \simeq \mathrm{HH}^{n-4}(R) \oplus \mathbb{Z}_{2}^{8} .
$$

Proof. By Proposition 2.7 we have the following short exact sequences of complexes:

$$
0 \rightarrow \operatorname{Hom}_{\Lambda}\left(Q_{\bullet}[-4], R\right) \stackrel{\pi^{*}}{\longrightarrow} \operatorname{Hom}_{\Lambda}\left(Q_{\bullet}, R\right) \stackrel{i^{*}}{\longrightarrow} \mathcal{X} \bullet 0,
$$

where $\mathcal{X}^{\bullet}=\operatorname{Hom}_{\Lambda}\left(X_{\bullet}, R\right)$. In its turn, this leads to the long exact cohomology sequence

$$
\ldots \stackrel{\Delta^{n-1}}{\longrightarrow} \mathrm{HH}^{n-4}(R) \stackrel{\pi^{*}}{\longrightarrow} \mathrm{HH}^{n}(R) \stackrel{\imath^{*}}{\longrightarrow} \mathrm{H}^{n}\left(\mathcal{X} \bullet \stackrel{\Delta^{n}}{\longrightarrow} \mathrm{HH}^{n-3}(R) \stackrel{\pi^{*}}{\longrightarrow} \cdots\right.
$$

Now, we need to compute the cohomology for the auxiliary complex $\mathcal{X}^{\bullet}$.

Lemma 3.23. For any $n \geq 4$, we have $\mathrm{H}^{n}\left(\mathcal{X}^{\bullet}\right) \simeq \mathbb{Z}_{2}^{8}$.

Proof. Observe that $\mathcal{X}^{n} \simeq R^{4}$ for $n \geq 3$. Hence, as at the beginning of this section, we may identify a cochain in $\mathcal{X}^{n}$ with the collection of its values at the corresponding generators. Also, we note that the complex $\mathcal{X}^{\bullet}$ is 2-periodic starting with the term $\mathcal{X}^{3}$.

a) If $n$ is odd, then the condition $\left(r_{1}, r_{2}, r_{3}, r_{4}\right) \in \operatorname{Ker} \delta_{\mathcal{X}}^{n}$ is equivalent to the system of equations obtained from (3.37) by omitting the first equation. Arguing as at the beginning of the proof of Proposition [3.14, we may assume that $r_{3}=0=r_{4}$. Then $B^{-} * r_{1}=0$ and $C^{-} * r_{2}=0$. As in the proof of Proposition 3.9. we see that $\operatorname{Ker} \delta_{\mathcal{X}}^{n}$ has a basis formed by the elements

$$
\begin{aligned}
& \left(1, \mathrm{O}_{3}\right),\left(b, \mathrm{O}_{3}\right),\left(c(b c)^{m-1}, \mathrm{O}_{3}\right),\left((b c)^{m}, \mathrm{O}_{3}\right) \\
& \left(0,1, \mathrm{O}_{2}\right),\left(0, c, \mathrm{O}_{2}\right),\left(0, b(c b)^{m-1}, \mathrm{O}_{2}\right),\left(0,(b c)^{m}, \mathrm{O}_{2}\right) \\
& \left((b c)^{i}+(c b)^{i}, \mathrm{O}_{3}\right), \quad\left(c(b c)^{i-1}+b(c b)^{i}, \mathrm{O}_{3}\right), \quad 1 \leq i \leq m-1 ; \\
& \left(0,(b c)^{i}+(c b)^{i}, \mathrm{O}_{2}\right), \quad\left(0, b(c b)^{i-1}+c(b c)^{i}, \mathrm{O}_{2}\right), 1 \leq i \leq m-1 .
\end{aligned}
$$

We can verify directly (cf. the proof of Proposition 3.8) that $\operatorname{Im} \delta_{\mathcal{X}}^{n-1}$ admits the following basis:

$$
\begin{aligned}
& \left(2, \mathrm{O}_{3}\right),\left(2, \mathrm{O}_{3}\right),\left(2 c(b c)^{m-1}, \mathrm{O}_{3}\right),\left(2(b c)^{m}, \mathrm{O}_{3}\right) \\
& \left(0,2, \mathrm{O}_{2}\right),\left(0,2 c, \mathrm{O}_{2}\right),\left(0,2 b(c b)^{m-1}, \mathrm{O}_{2}\right), \quad\left(0,2(b c)^{m}, \mathrm{O}_{2}\right) \\
& \left((b c)^{i}+(c b)^{i}, \mathrm{O}_{3}\right), \quad\left(c(b c)^{i-1}+b(c b)^{i}, \mathrm{O}_{3}\right), 1 \leq i \leq m-1 \\
& \left(0,(b c)^{i}+(c b)^{i}, \mathrm{O}_{2}\right), \quad\left(0, b(c b)^{i-1}+c(b c)^{i}, \mathrm{O}_{2}\right), 1 \leq i \leq m-1 .
\end{aligned}
$$


Since these bases of the $\mathbb{Z}$-modules $\operatorname{Im} \delta_{\mathcal{X}}^{n-1}$ and $\operatorname{Ker} \delta_{\mathcal{X}}^{n}$ are stacked, the desired statement follows. Moreover, the cohomology classes of the elements in (3.75)-(3.76) form a set of generators for $\mathrm{H}^{n}\left(\mathcal{X}^{\bullet}\right)$, and the order of every element of this set in the group $\mathrm{H}^{n}\left(\mathcal{X}^{\bullet}\right)$ is equal to 2 .

b) In the case where $n$ is even, the proof is similar. In this case we can take a generating set for $\mathrm{H}^{n}\left(\mathcal{X}^{\bullet}\right)$ formed by the cohomology classes of the elements

$$
\begin{aligned}
& \left(\mathrm{O}_{2}, 1,0\right),\left(\mathrm{O}_{2}, b, 0\right),\left(\mathrm{O}_{2}, c(b c)^{m-1}, 0\right),\left(\mathrm{O}_{2},(b c)^{m}, 0\right) \\
& \left(\mathrm{O}_{3}, 1\right),\left(\mathrm{O}_{3}, c\right),\left(\mathrm{O}_{3}, b(c b)^{m-1}\right),\left(\mathrm{O}_{3},(b c)^{m}\right) .
\end{aligned}
$$

The order of each class in this set is equal to 2 .

We continue the proof of Proposition 3.22 First, we prove that the connecting homomorphisms $\Delta^{n}$ in the sequence (3.74) are zero if $n \geq 4$.

Case 1: Assume that $n=2 k+1$ with $k \in \mathbb{N}, k \geq 2$. We consider a $(2 k+1)$-cocycle $f=\left(r_{1}, r_{2}, r_{3}, r_{4}\right) \in \mathcal{X}^{2 k+1}$, i.e. $\delta_{\mathcal{X}}^{2 k+1}(f)=0$. By the description of the generating set for $\mathrm{H}^{2 k+1}\left(\mathcal{X}^{\bullet}\right)$ given in the proof of Lemma 3.23 (see (3.75) and (3.76) ), we may assume that $r_{3}=0=r_{4}$. Then we also have $B^{-} * r_{1}=0=C^{-} * r_{2}$.

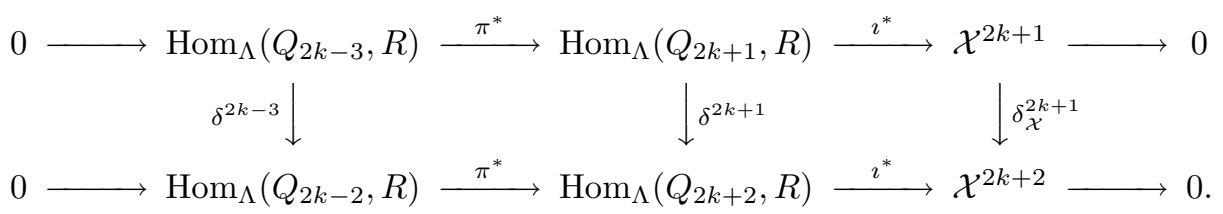

Define $\tilde{f}=f \circ \nu$, where $\nu: Q_{2 k+1} \rightarrow X_{2 k+1}$ is the projection onto the corresponding direct summand. In other words, the map $\tilde{f}$ is obtained from $f$ via extension by zero to the direct summand complementary to $X_{2 k+1}$ in $Q_{2 k+1}$; thus, $\widetilde{f}=\left(\mathrm{O}_{2 k-2}, r_{1}, r_{2}, 0,0\right)$. Consequently, $i^{*}(\tilde{f})=\tilde{f} \circ i=f \circ \nu \circ i=f$. By the construction of the connecting homomorphism, we have $\Delta^{2 k+1}(\operatorname{cl} f)=\operatorname{cl} g$, where $g: Q_{2 k-2} \rightarrow R$ is a cocycle for which $\pi^{*}(g)=\delta^{2 k+1} \tilde{(f)}$.

Consider the cochain $\varphi=\left(\mathrm{O}_{2 k-4}, r_{1} \cdot(b c)^{m},-r_{2} \cdot(b c)^{m}\right) \in \operatorname{Hom}_{\Lambda}\left(Q_{2 k-3}, R\right)$. If $k \geq 3$, then by Lemma 3.10 .

$$
\begin{aligned}
\delta^{2 k-3}(\varphi) & =\left(\mathrm{O}_{2 k-5},-\rho^{-} * r_{1}(b c)^{m},-\omega^{-} * \rho_{2}(b c)^{m}, B^{+} * r_{1}(b c)^{m},-C^{+} * r_{2}(b c)^{m}\right) \\
& =\left(\mathrm{O}_{2 k-3}, \rho^{+} * r_{1},-\omega^{+} * r_{2}\right) .
\end{aligned}
$$

Consequently, $\pi^{*}\left(\delta^{2 k-3}(\varphi)\right)=\delta^{2 k+1}(\widetilde{f})$. Putting $g=\delta^{2 k-3}(\varphi)$ we obtain $\Delta^{2 l+1}(\operatorname{cl} f)=$ $\operatorname{cl} g=0$.

Before studying the case where $k=2$, we prove the following auxiliary statement.

Lemma 3.24. Let $f_{\zeta^{-}}, f_{\eta^{-}}: R \rightarrow R$ be homomorphisms such that $f_{\zeta^{-}}(r)=\zeta^{-} * r$ and $f_{\eta^{-}}(r)=\eta^{-} * r$, and let $f_{b}^{-}, f_{c}^{-}: R \rightarrow R$ be the homomorphisms occurring in Lemma 3.15. Then
a) $\operatorname{Ker} f_{b}^{-} \subset \operatorname{Ker} f_{\zeta^{-}}$
b) $\operatorname{Ker} f_{c}^{-} \subset \operatorname{Ker} f_{\eta^{-}}$. 
Proof. a) For any $r \in \operatorname{Ker} f_{b}^{-}$, we have

$$
\begin{aligned}
\sum_{i=0}^{m-1} c(b c)^{i} \cdot r \cdot(c b)^{m-1-i} & =\sum_{i=0}^{m-1} c(b c)^{i}(c b)^{m} \cdot r \cdot(c b)^{-1-i}=\sum_{i=0}^{m-1}(b c)^{m-1-i} b \cdot r \cdot(b c)^{1+i} \\
& =\sum_{i=0}^{m-1}(b c)^{m-1-i} \cdot r \cdot b(b c)^{1+i}=\sum_{j=0}^{m-1}(b c)^{j} \cdot r \cdot(c b)^{m-1-j} c
\end{aligned}
$$

(in the latter identity we have changed the summation: $j=m-1-i$ ). Consequently, $\zeta^{-} * r=0$.

Part b) follows from a) by symmetry.

We continue the proof of Proposition 3.22 Assume that $k=2$, i.e., $n=5$. For the 1-cochain $\varphi=\left(r_{1} \cdot(b c)^{m},-r_{2} \cdot(b c)^{m}\right)$, which has already been used, Lemma 3.24 shows that

$$
\begin{aligned}
\delta^{1}(\varphi) & =\left(-\zeta^{-} * r_{1}(b c)^{m}-\eta^{-} * r_{2}(b c)^{m}, B^{+} * r_{1}(b c)^{m},-C^{+} * r_{2}(b c)^{m}\right) \\
& =\left(0, \rho^{+} * r_{1},-\omega^{+} * r_{2}\right) .
\end{aligned}
$$

Putting again $g=\delta^{1}(\varphi)$, we obtain $\Delta^{5}(\operatorname{cl} f)=\operatorname{cl} g=0$.

Case 2: Assume that $n=2 k$ with $k \in \mathbb{N}, k \geq 2$.

Lemma 3.25. a) If $B^{+} * r+\rho^{-} * s=0$ with $r, s \in R$, then $B^{+} * r=0=\rho^{-} * s$.

b) If $C^{+} * r+\omega^{-} * s=0$ with $r, s \in R$, then $C^{+} * r=0=\omega^{-} * s$.

Proof. a) Since

$$
2(r+b r b)=B^{+} *\left(B^{+} * r\right)=-B^{+} *\left(\rho^{-} * r\right)=-\left(B^{+} \rho^{-}\right) * r=0,
$$

we obtain $b r+r b=b(r+b r b)=0$.

Part b) follows from a) by symmetry.

We continue the proof of Proposition 3.22. Consider a $2 k$-cocycle

$$
f \in \mathcal{X}^{2 k}=\left(r_{1}, r_{2}, r_{3}, r_{4}\right) \in \operatorname{Hom}_{\Lambda}\left(X_{2 k}, R\right),
$$

i.e., $\delta_{\mathcal{X}}^{2 k}(f)=0$. Using Lemma 3.25 we see, in particular, that $B^{+} * r_{1}=0=C^{+} * r_{2}$. Set $\tilde{f}=f \circ \nu$, where $\nu: Q_{2 k} \rightarrow X_{2 k}$ is the projection onto the corresponding direct summand. Then $\delta^{2 k}(\widetilde{f})=\left(\mathrm{O}_{2 k-4},-\rho^{+} * r_{1}, \omega^{+} * r_{1}, \mathrm{O}_{4}\right)=0$, whence $\Delta^{2 k}(\mathrm{cl} f)=0$.

Consequently, for any $n \geq 5$ we have the short exact sequence

$$
0 \rightarrow \mathrm{HH}^{n-4}(R) \stackrel{\pi^{*}}{\longrightarrow} \mathrm{HH}^{n}(R) \stackrel{\imath^{*}}{\longrightarrow} \mathrm{H}^{n}\left(\mathcal{X}^{\bullet}\right) \rightarrow 0 .
$$

Since $\mathrm{HH}^{1}(R)=0$ (see Proposition 3.7), the sequence (3.77) and Lemma 3.23 show that $\mathrm{HH}^{5}(R) \simeq \mathbb{Z}_{2}^{8}$. Moreover, (3.77) yields the following identity:

$$
\left|\mathrm{HH}^{n}(R)\right|=\left|\mathrm{HH}^{n-4}(R)\right|+2^{8} .
$$

Now we assume that $n$ is odd: $n=2 l+1$ with $l \geq 3$. Observe that the matrix of the differential $d_{2 l+1}^{Q}$ has the following block structure:

$$
d_{2 l+1}^{Q}=\left(\begin{array}{cc|cc}
d_{2 l-3}^{Q} & \mathrm{O}_{2 l-2,4} \\
\hline \mathrm{O}_{2,2 l-3} & \sigma^{+} & D^{-} & \mathrm{O} \\
\mathrm{O}_{2,2 l-3} & \mathrm{O} & -\sigma^{-} & D^{+}
\end{array}\right) ;
$$


we have used the abbreviated notation (2.4) and (2.5). It is easily seen that the following cochains in $\operatorname{Hom}_{\Lambda}\left(Q_{2 l+1}, R\right)$ are cocycles:

$$
\begin{array}{ll}
f_{1}:=\left(\mathrm{O}_{2 l-4},-(b c)^{m}, 0,1, \mathrm{O}_{3}\right), & f_{2}:=\left(\mathrm{O}_{2 l-4},-c(b c)^{m-1}, 0, b, \mathrm{O}_{3}\right), \\
f_{3}:=\left(\mathrm{O}_{2 l-4},-b, 0, c(b c)^{m-1}, \mathrm{O}_{3}\right), & f_{4}:=\left(\mathrm{O}_{2 l-4},-1,0,(b c)^{m}, \mathrm{O}_{3}\right), \\
f_{5}:=\left(\mathrm{O}_{2 l-3},(b c)^{m}, 0,1, \mathrm{O}_{2}\right), & f_{6}:=\left(\mathrm{O}_{2 l-3}, b(c b)^{m-1}, 0, c, \mathrm{O}_{2}\right), \\
f_{7}:=\left(\mathrm{O}_{2 l-3}, c, 0, b(c b)^{m-1}, \mathrm{O}_{2}\right), & f_{8}:=\left(\mathrm{O}_{2 l-3}, 1,0,(b c)^{m}, \mathrm{O}_{2}\right) .
\end{array}
$$

Let $L$ be a subgroup of $\mathrm{HH}^{2 l+1}(R)$ generated by the set $\left\{\operatorname{cl} f_{i}\right\}_{i=1}^{8}$. We shall prove that

$$
\mathrm{HH}^{2 l+1}(R)=\pi^{*}\left(\mathrm{HH}^{2 l-3}(R)\right)+L .
$$

We write any cocycle $x \in \operatorname{Ker} \delta^{2 l+1}$ in the form $x=\left(x^{\prime}, x^{\prime \prime}\right)$, where $x^{\prime} \in \operatorname{Hom}_{\Lambda}\left(Q_{2 l-3}, R\right)$ and $x^{\prime \prime} \in \operatorname{Hom}_{\Lambda}\left(X_{2 l+1}, R\right)$. By (3.79), we have $x^{\prime \prime}=\left(r_{1}, r_{2}, r_{3}, r_{4}\right) \in \operatorname{Ker} \delta_{\mathcal{X}}^{2 l+1}$. Since $D^{+}\left(r_{3}, r_{4}\right)=0$, Lemma 3.15 shows that $r_{3}=B^{-} * u$ and $r_{4}=C^{-} * u^{\prime}$ for some $u, u^{\prime} \in R$.

Replacing $x$ by $x-\delta^{2 l}\left(\mathrm{O}_{2 l-1}, u, u^{\prime}\right)$, we may assume that $r_{3}=r_{4}=0$ for the initial $x$. Subtracting from $x$ a suitable linear combination of elements of the set $\left\{f_{i}\right\}_{i=1}^{8}$ and using the arguments of part a) of the proof of Lemma 3.23, we get an element of the form $y=\left(y^{\prime}, \mathrm{O}_{4}\right) \in \operatorname{Ker} \delta^{2 l+1}$. Using (3.79), we see that $y^{\prime} \in \operatorname{Ker} \delta^{2 l-3}$. Hence, we have $y \in \operatorname{Im} \pi^{*}$, so that $x \in \operatorname{Im} \pi^{*}+L$. This completes the proof of (3.80).

Now, we observe that the matrix of the differential $d_{2 l}^{Q}$ has the following block structure:

$$
d_{2 l}^{Q}=\left(\begin{array}{cc|cc}
d_{2 l-4}^{Q} & \mathrm{O}_{2 l-3,4} \\
\hline \mathrm{O}_{2,2 l-4} & -\sigma^{+} & D^{+} & \mathrm{O} \\
\mathrm{O}_{2,2 l-4} & \mathrm{O} & \sigma^{-} & D^{-}
\end{array}\right) .
$$

Consider the following cochains in $\operatorname{Hom}_{\Lambda}\left(Q_{2 l}, R\right)$ :

$$
\begin{array}{ll}
g_{1}:=\left(\mathrm{O}_{2 l-3}, b, \mathrm{O}_{3}\right), & g_{2}:=\left(\mathrm{O}_{2 l-3}, 1, \mathrm{O}_{3}\right), \\
g_{3}:=\left(\mathrm{O}_{2 l-3},(b c)^{m}, \mathrm{O}_{3}\right), & g_{4}:=\left(\mathrm{O}_{2 l-3}, c(b c)^{m-1}, \mathrm{O}_{3}\right), \\
g_{5}:=\left(\mathrm{O}_{2 l-2}, c, \mathrm{O}_{2}\right), & g_{6}:=\left(\mathrm{O}_{2 l-2}, 1, \mathrm{O}_{2}\right), \\
g_{7}:=\left(\mathrm{O}_{2 l-2},(b c)^{m}, \mathrm{O}_{2}\right), & g_{8}:=\left(\mathrm{O}_{2 l-2}, b(c b)^{m-1}, \mathrm{O}_{2}\right) .
\end{array}
$$

Using (3.81), we easily check that $\delta^{2 l}\left(g_{i}\right)=2 f_{i}$ for all $i=1, \ldots, 8$. In particular, $|L| \leq 2^{8}$. Moreover, (3.80) implies the existense of an epimorphism $\mathrm{HH}^{2 l-3}(R) \oplus L \rightarrow \mathrm{HH}^{2 l+1}(R)$. Then, by (3.78), this epimorphism is an isomorphism and $L \simeq \mathbb{Z}_{2}^{8}$.

Finally, we study the groups $\operatorname{HH}^{n}(R)$ for even $n$ : let $n=2 l$ with $l \geq 3$. It is easily verified that the following cochains in $\operatorname{Hom}_{\Lambda}\left(Q_{2 l}, R\right)$ are cocycles:

$$
\begin{array}{ll}
f_{1}:=\left(\mathrm{O}_{2 l-1}, 1,0\right), & f_{2}:=\left(\mathrm{O}_{2 l-1}, b, 0\right), \\
f_{3}:=\left(\mathrm{O}_{2 l-1}, c(b c)^{m-1}, 0\right), & f_{4}:=\left(\mathrm{O}_{2 l-1},(b c)^{m}, 0\right), \\
f_{5}:=\left(\mathrm{O}_{2 l}, 1\right), & f_{6}:=\left(\mathrm{O}_{2 l}, c\right), \\
f_{7}:=\left(\mathrm{O}_{2 l}, b(c b)^{m-1}\right), & f_{8}:=\left(\mathrm{O}_{2 l},(b c)^{m}\right) .
\end{array}
$$


Let $L$ be the subgroup of $\operatorname{HH}^{2 l}(R)$ generated by the $\operatorname{set}\left\{\operatorname{cl} f_{i}\right\}_{i=1}^{8}$. Arguing as above, we check that $\mathrm{HH}^{2 l}(R)=\pi^{*}\left(\mathrm{HH}^{2 l-4}(R)\right)+L$. The cochains

$$
\begin{array}{ll}
g_{1}:=\left(\mathrm{O}_{2 l-1}, b, 0\right), & g_{2}:=\left(\mathrm{O}_{2 l-1}, 1,0\right), \\
g_{3}:=\left(\mathrm{O}_{2 l-1},(b c)^{m}, 0\right), & g_{4}:=\left(\mathrm{O}_{2 l-1}, c(b c)^{m-1}, 0\right), \\
g_{5}:=\left(\mathrm{O}_{2 l}, c\right), & g_{6}:=\left(\mathrm{O}_{2 l}, 1\right), \\
g_{7}:=\left(\mathrm{O}_{2 l},(b c)^{m}\right), & g_{8}:=\left(\mathrm{O}_{2 l}, b(c b)^{m-1}\right)
\end{array}
$$

satisfy the relations $\delta^{2 l-1}\left(g_{i}\right)=2 f_{i}$ for all $i=1, \ldots, 8$. As above, we conclude that $\mathrm{HH}^{2 l}(R)=\mathrm{HH}^{2 l-4}(R) \oplus L$ and $L \simeq \mathbb{Z}_{2}^{8}$.

Corollary 3.26. The group $\mathrm{HH}^{5}(R)$ is generated by the cohomology classes of the following elements:

$$
\begin{array}{ll}
t_{1}:=\left(-(b c)^{m}, 0,1, \mathrm{O}_{3}\right), & t_{2}:=\left(-c(b c)^{m-1}, 0, b, \mathrm{O}_{3}\right), \\
t_{3}:=\left(-b, 0, c(b c)^{m-1}, \mathrm{O}_{3}\right), & t_{4}:=\left(-1,0,(b c)^{m}, \mathrm{O}_{3}\right), \\
t_{1}^{\prime}:=\left(0,(b c)^{m}, 0,1, \mathrm{O}_{2}\right), & t_{2}^{\prime}:=\left(0, b(c b)^{m-1}, 0, c, \mathrm{O}_{2}\right), \\
t_{3}^{\prime}:=\left(0, c, 0, b(c b)^{m-1}, \mathrm{O}_{2}\right), & t_{4}^{\prime}:=\left(0,1,0,(b c)^{m}, \mathrm{O}_{2}\right),
\end{array}
$$

which satisfy the relations

$$
2 t_{j}=2 t_{j}^{\prime}=0 \text { for } 1 \leq j \leq 4 .
$$

Proof. Clearly, the images of the cohomology classes of the elements (3.82)-(3.85) under the map $i^{*}: \mathrm{HH}^{5}(R) \rightarrow \mathrm{HH}^{5}(\mathcal{X})$ generate the group $\mathrm{HH}^{5}(\mathcal{X})$ (see the elements (3.75) and (3.76) indicated in the proof of Lemma 3.23). Since $i^{*}$ is an isomorphism, the corollary follows.

Proof of Proposition 1.4. The statement follows by induction from Proposition 3.22 with the help of Propositions 3.1, 3.7, 3.11, 3.16, and 3.21,

\section{§4. Generators and Relations}

We recall briefly an interpretation of the Yoneda product in the algebra $\operatorname{HH}^{*}(R)=$ $\bigoplus_{n \geq 0} \operatorname{Ext}_{\Lambda}^{n}(R, R)$, which was used ealier in [7, 8. Let $\mu: Q \bullet \rightarrow R$ be the $\Lambda$-free resolution constructed in $\S 2$. Let $\operatorname{Hom}_{\Lambda}\left(Q_{\bullet}, R\right)=\left(\operatorname{Hom}_{\Lambda}\left(Q_{n}, R\right), \delta^{n}\right)$ be the complex (3.1). Any $n$-cocycle $f \in \operatorname{Ker} \delta^{n}$ is lifted (uniquely up to homotopy) to a chain map of complexes $\left\{\varphi_{i}: Q_{n+i} \rightarrow Q_{i}\right\}_{i \geq 0}$. The homomorphism $\varphi_{i}$ is called the $i$ th translate of the cocycle $f$ and will be denoted by $\mathrm{T}^{i}(f)$. For cocycles $f \in \operatorname{Ker} \delta^{n}$ and $g \in \operatorname{Ker} \delta^{t}$ we have $\operatorname{cl} g \cdot \operatorname{cl} f=\operatorname{cl}\left(\mu \mathrm{T}^{0}(g) \mathrm{T}^{t}(f)\right)$. Consequently, it suffices to know the composition of the translates $\mathrm{T}^{0}(g)$ and $\mathrm{T}^{t}(f)$. In the sequel, using direct decompositions of the modules $Q_{i} \simeq \Lambda^{i+1}$, we shall describe translates of cocycles and their products in terms of matrices that correspond to such decompositions (cf. the description of the differentials $d_{n}^{Q}$ in 93 ). Observe that, by the graded commutativity of the algebra $\mathrm{HH}^{*}(R)$, in order to compute the product of a pair of different homogeneous elements in $\mathrm{HH}^{*}(R)$, it suffices to know, for an element of degree $i$, its translates of order not exceeding $i$. 
We denote by $\mathcal{Y}$ the set formed by the following homogeneous elements of $\mathrm{HH}^{*}(R)$ (these elements occurred in the description of the groups $\mathrm{HH}^{i}(R)$ with $i \leq 4$; see 93 ):

—of degree $0: \quad p_{1}:=\theta_{b}, p_{1}^{\prime}:=\theta_{c}, p_{2}:=b c+c b, p_{3}:=(b c)^{m} ;$

— of degree 2: $\quad u_{0}:=u_{0}^{(1)}:=\left(b c-c b, \mathrm{O}_{2}\right), u_{1}:=(0,1,0), u_{1}^{\prime}:=\left(\mathrm{O}_{2}, 1\right)$,

$$
u_{2}:=(0, b, 0), u_{2}^{\prime}:=\left(\mathrm{O}_{2}, c\right)
$$

—of degree 3: $\quad v:=v_{1}:=\left(b, c, \mathrm{O}_{2}\right)$;

—of degree $4: \quad w:=w_{1}:=\left(1, \mathrm{O}_{4}\right)$.

Remark 4.1. By Proposition 2.7, there is a chain map $\pi: Q_{\bullet} \rightarrow Q_{\bullet}[-4]$ for which $\pi_{n}: Q_{n}=Q_{n-4} \oplus X_{n} \rightarrow Q_{n-4}$ is the projection onto the corresponding direct summand. Consequently, $w$ coincides with $\mu \pi_{4}$ (here, $\mu: Q_{0}=\Lambda \rightarrow R$ is the augmentation map of the resolution; see Remark 2.3); moreover, we can take the following translates of the cocycle $w: \mathrm{T}^{i}(w)=\pi_{i+4}(i \geq 0)$.

Proposition 4.2. For the role of translates of the elements in $\mathcal{Y} \backslash\{w\}$ that have positive degree, we can take the homomorphisms determined by the following matrices:

$$
\begin{aligned}
& \mathrm{T}^{0}\left(u_{0}\right)=(b \otimes c-c \otimes b, 0,0), \quad \mathrm{T}^{1}\left(u_{0}\right)=\left(\begin{array}{cccc}
0 & 1 \otimes 1+c \otimes c & 0 & 0 \\
-1 \otimes 1-b \otimes b & 0 & 0 & 0
\end{array}\right), \\
& \mathrm{T}^{2}\left(u_{0}\right)=\left(\begin{array}{ccccc}
c b \otimes 1-b c \otimes 1 & 0 & 0 & 0 & 0 \\
\star & 0 & 0 & 0 & 0 \\
\star & 0 & 0 & 0 & 0
\end{array}\right) \text {, }
\end{aligned}
$$

with

$$
\begin{aligned}
& \mathrm{T}^{2}\left(u_{0}\right)_{2,1}=-\sum_{i=0}^{m-2} b(c b)^{i} \otimes c(b c)^{m-2-i}-\sum_{i=0}^{m-1}(c b)^{i} \otimes(c b)^{m-1-i}-c \otimes c(b c)^{m-1} \\
& \mathrm{~T}^{2}\left(u_{0}\right)_{3,1}=-\sum_{i=0}^{m-2} c(b c)^{i} \otimes b(c b)^{m-2-i}-\sum_{i=0}^{m-1}(b c)^{i} \otimes(b c)^{m-1-i}-b \otimes b(c b)^{m-1}
\end{aligned}
$$

at the positions $(2,1)$ and $(3,1)$ respectively;

$$
\begin{aligned}
& \mathrm{T}^{0}\left(u_{1}\right)=(0,1 \otimes 1,0), \quad \mathrm{T}^{1}\left(u_{1}\right)=\left(\begin{array}{llll}
\sum_{i=0}^{m-2} c(b c)^{i} \otimes c(b c)^{m-2-i} & 0 & 1 \otimes 1 & 0 \\
\sum_{i=0}^{m-1}(c b)^{i} \otimes(b c)^{m-1-i} & 0 & 0 & 0
\end{array}\right), \\
& \mathrm{T}^{2}\left(u_{1}\right)=\left(\begin{array}{ccccc}
0 & 1 \otimes 1 & 0 & 0 & 0 \\
\star & 0 & 0 & 1 \otimes 1 & 0 \\
\star & 0 & 0 & 0 & 0
\end{array}\right)
\end{aligned}
$$

with

$$
\begin{aligned}
\mathrm{T}^{2}\left(u_{1}\right)_{2,1} & =\sum_{i=0}^{m-2}(m-1-i)(b c)^{i} \otimes(c b)^{i}+\sum_{i=0}^{m-2}(m-1-i) c(b c)^{i} \otimes c(b c)^{i}, \\
\mathrm{~T}^{2}\left(u_{1}\right)_{3,1} & =\sum_{i=0}^{m-1}(m-i)(c b)^{i} \otimes(b c)^{i}+\sum_{i=1}^{m-1}(m-i) b(c b)^{i-1} \otimes b(c b)^{i-1}
\end{aligned}
$$




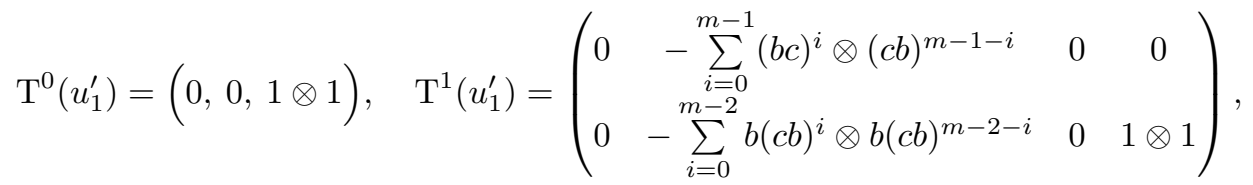

$$
\begin{aligned}
& \mathrm{T}^{2}\left(u_{1}^{\prime}\right)=\left(\begin{array}{ccccc}
0 & 0 & 1 \otimes 1 & 0 & 0 \\
\star & 0 & 0 & 0 & 0 \\
\star & 0 & 0 & 0 & 1 \otimes 1
\end{array}\right)
\end{aligned}
$$

with

$$
\begin{aligned}
& \mathrm{T}^{2}\left(u_{1}^{\prime}\right)_{2,1}=\sum_{i=0}^{m-1}(m-i)(b c)^{i} \otimes(c b)^{i}+\sum_{i=1}^{m-1}(m-i) c(b c)^{i-1} \otimes c(b c)^{i-1}, \\
& \mathrm{~T}^{2}\left(u_{1}^{\prime}\right)_{3,1}=\sum_{i=0}^{m-2}(m-1-i)(c b)^{i} \otimes(b c)^{i}+\sum_{i=0}^{m-2}(m-1-i) b(c b)^{i} \otimes b(c b)^{i} ; \\
& \mathrm{T}^{0}\left(u_{2}\right)=(0, b \otimes 1,0), \quad \mathrm{T}^{1}\left(u_{2}\right)=\left(\begin{array}{llcc}
\sum_{i=1}^{m-1}(b c)^{i} \otimes c(b c)^{m-1-i} & 0 & b \otimes 1 & 0 \\
\sum_{i=0}^{m-1} b(c b)^{i} \otimes(b c)^{m-1-i} & 0 & 0 & 0
\end{array}\right), \\
& \mathrm{T}^{2}\left(u_{2}\right)=\left(\begin{array}{ccccc}
0 & b \otimes 1 & 0 & 0 & 0 \\
\star & 0 & 0 & b \otimes 1 & 0 \\
\star & 0 & 0 & 0 & 0
\end{array}\right)
\end{aligned}
$$

with

$$
\begin{aligned}
& \mathrm{T}^{2}\left(u_{2}\right)_{2,1}=\sum_{i=0}^{m-2} \sum_{j=0}^{m-1-i}(b c)^{i+2 j} \otimes b(c b)^{i}+\sum_{i=1}^{m-1} \sum_{j=0}^{m-1-i} c(b c)^{i+1+2 j} \otimes(b c)^{i}, \\
& \mathrm{~T}^{2}\left(u_{2}\right)_{3,1}=\sum_{i=1}^{m-1} \sum_{j=0}^{m-1-i} b(c b)^{i+2 j} \otimes(c b)^{i}+\sum_{i=0}^{m-1} \sum_{j=0}^{m-1-i}(c b)^{i+1+2 j} \otimes c(b c)^{i} ; \\
& \mathrm{T}^{0}\left(u_{2}^{\prime}\right)=(0,0, c \otimes 1), \quad \mathrm{T}^{1}\left(u_{2}^{\prime}\right)=\left(\begin{array}{cccc}
0 & -\sum_{i=1}^{m-1} c(b c)^{i} \otimes(c b)^{m-1-i} & 0 & 0 \\
0 & -\sum_{i=0}^{m-1}(c b)^{i} \otimes b(c b)^{m-1-i} & 0 & c \otimes 1
\end{array}\right), \\
& \mathrm{T}^{2}\left(u_{2}^{\prime}\right)=\left(\begin{array}{ccccc}
0 & 0 & c \otimes 1 & 0 & 0 \\
\star & 0 & 0 & 0 & 0 \\
\star & 0 & 0 & 0 & c \otimes 1
\end{array}\right)
\end{aligned}
$$

with

$$
\begin{aligned}
\mathrm{T}^{2}\left(u_{2}^{\prime}\right)_{2,1} & =\sum_{i=1}^{m-1} \sum_{j=0}^{m-1-i} c(b c)^{i+2 j} \otimes(b c)^{i}+\sum_{i=0}^{m-1} \sum_{j=0}^{m-1-i}(b c)^{i+1+2 j} \otimes b(c b)^{i}, \\
\mathrm{~T}^{2}\left(u_{2}^{\prime}\right)_{3,1} & =\sum_{i=0}^{m-2} \sum_{j=1}^{m-1-i}(c b)^{i+2 j} \otimes c(b c)^{i}+\sum_{i=1}^{m-1} \sum_{j=0}^{m-1-i} b(c b)^{i+1+2 j} \otimes(c b)^{i}
\end{aligned}
$$


$\mathrm{T}^{0}(v)=(b \otimes 1, c \otimes 1,0,0), \quad \mathrm{T}^{1}(v)=\left(\begin{array}{lllll}\sum_{i=0}^{m-1} c(b c)^{i} \otimes(c b)^{m-1-i} & b \otimes 1 & 0 & 0 & 0 \\ -\sum_{i=0}^{m-1} b(c b)^{i} \otimes(b c)^{m-1-i} & 0 & c \otimes 1 & 0 & 0\end{array}\right)$, $\mathrm{T}^{2}(v)=\left(\begin{array}{cccccc}-b \otimes 1 & -c \otimes 1 & 0 & 0 & 0 & 0 \\ -c(b c)^{m-1} \otimes 1 & 0 & b \otimes 1 & 0 & 0 & 0 \\ 0 & b\left(c b^{m-1} \otimes 1\right. & 0 & c \otimes 1 & 0 & 0\end{array}\right)$,

$\mathrm{T}^{3}(v)=t\left(\begin{array}{ccccccc}-\sum_{i=0}^{m-1}(b c)^{i} \otimes c(b c)^{m-1-i} & -b \otimes 1 & 0 & 0 & 0 & 0 & 0 \\ \sum_{i=0}^{m-1}(c b)^{i} \otimes b(c b)^{m-1-i} & 0 & -c \otimes 1 & 0 & 0 & 0 & 0 \\ 0 & c(b c)^{m-1} \otimes 1 & 0 & b \otimes 1 & 0 & 0 & 0 \\ 0 & 0 & -b(c b)^{m-1} \otimes 1 & 0 & c \otimes 1 & 0 & 0\end{array}\right)$.

Proof. This proposition is proved by direct verification of the relations $\mu \mathrm{T}^{0}(x)=x$, $d_{i-1} \mathrm{~T}^{i}(x)=\mathrm{T}^{i-1}(x) d_{i+\operatorname{deg} x}(0<i \leq \operatorname{deg} x)$, where $x \in \mathcal{Y} \backslash\{w\}$ with $\operatorname{deg} x>0$, and we leave this to the reader.

We introduce an auxiliary notation:

$$
q_{i}:=(b c)^{i}+(c b)^{i} \in Z(R) \text { for } 1 \leq i \leq m
$$

(clearly, $q_{1}=p_{2}, q_{m}=2 p_{3}$ ).

The following statement is deduced immediately from the definitions.

Lemma 4.3. Let $f_{i}$ with $i \in \mathbb{N}$ be the polynomials defined by (1.3).

a) For any $1 \leq i \leq m$, we have $q_{i}=f_{i}\left(p_{2}\right)$.

b) For any $1 \leq i \leq m$, we have

$$
p_{2}^{i}= \begin{cases}\mathrm{C}_{i}^{\frac{i}{2}} \cdot 1+\sum_{t=0}^{\left[\frac{i-1}{2}\right]} \mathrm{C}_{i}^{t} q_{i-2 t} & \text { if } i \text { is even }, \\ \sum_{t=0}^{\left[\frac{i-1}{2}\right]} \mathrm{C}_{i}^{t} q_{i-2 t} & \text { if } i \text { is odd. }\end{cases}
$$

Remark 4.4. Lemma 4.3 implies that the subset $\left\{q_{i}\right\}_{i=1}^{m-1}$ of the basis of the group $\mathrm{HH}^{0}(R) \simeq Z(R)$ that was indicated in (3.4) can be replaced by the set $\left\{p_{2}^{i}\right\}_{i=1}^{m-1}$. Furthermore, we have $q_{i}=p_{2}^{i}+g_{i}\left(p_{2}\right)(1 \leq i \leq m)$.

Proposition 4.5. In the algebra $\mathrm{HH}^{*}(R)$, the elements of the set

$$
\mathcal{Y}=\left\{p_{1}, p_{1}^{\prime}, p_{2}, p_{3}, u_{0}, u_{1}, u_{1}^{\prime}, u_{2}, u_{2}^{\prime}, v, w\right\}
$$

satisfy the following relations:

$$
\begin{aligned}
& p_{1}^{2}=\left(p_{1}^{\prime}\right)^{2}= \begin{cases}m\left(1+\sum_{i=1}^{\left[\frac{m-1}{2}\right]} q_{2 i}\right) & \text { if } m \text { is odd }, \\
m\left(1+p_{3}+\sum_{i=1}^{\left.\frac{m-1}{2}\right]} q_{2 i}\right) & \text { if } m \text { is even } ;\end{cases} \\
& p_{1} p_{1}^{\prime}= \begin{cases}m\left(p_{3}+\sum_{i=1}^{\left[\frac{m}{2}\right]} q_{2 i-1}\right) & \text { if } m \text { is odd, } \\
m \sum_{i=1}^{\left[\frac{m}{2}\right]} q_{2 i-1} & \text { if } m \text { is even; }\end{cases}
\end{aligned}
$$




$$
\begin{aligned}
& p_{1} p_{3}=\left\{\begin{array}{ll}
p_{1}^{\prime} & \text { if } m \text { is odd }, \\
p_{1} & \text { if } m \text { is even; }
\end{array} \quad p_{1}^{\prime} p_{3}= \begin{cases}p_{1} & \text { if } m \text { is odd }, \\
p_{1}^{\prime} & \text { if } m \text { is even; }\end{cases} \right. \\
& p_{1} p_{2}=2 p_{1}^{\prime}, p_{1}^{\prime} p_{2}=2 p_{1}, p_{2} p_{3}=q_{m-1}, p_{3}^{2}=1 ; \\
& p_{1} u_{0}=p_{1}^{\prime} u_{0}=0 ;
\end{aligned}
$$$$
\left(1+\sum_{i=0}^{\left[\frac{m-2}{2}\right]} q_{m-1-2 i}\right) u_{0}=0 \text { if } m \text { is odd, }
$$$$
\sum_{i=0}^{\left[\frac{m-2}{2}\right]} q_{m-1-2 i} \cdot u_{0}=0 \text { if } m \text { is even; }
$$

$$
\begin{aligned}
& p_{3} u_{0}= \begin{cases}-\left(\sum_{i=0}^{\left[\frac{m-3}{2}\right]} q_{m-2-2 i}\right) u_{0} & \text { if } m \text { is odd }, \\
-\left(1+\sum_{i=0}^{\left[\frac{m-3}{2}\right]} q_{m-2-2 i}\right) u_{0} & \text { if } m \text { is even } ;\end{cases} \\
& p_{1} u_{1}=\left\{\begin{array}{ll}
u_{2} & \text { if } m \text { is odd }, \\
u_{2}+p_{3} u_{2} & \text { if } m \text { is even; }
\end{array} p_{1}^{\prime} u_{1}= \begin{cases}p_{3} u_{2} & \text { if } m \text { is odd }, \\
0 & \text { if } m \text { is even; }\end{cases} \right.
\end{aligned}
$$$$
p_{1} u_{1}^{\prime}=\left\{\begin{array}{ll}
p_{3} u_{2}^{\prime} & \text { if } m \text { is odd }, \\
0 & \text { if } m \text { is even } ;
\end{array} \quad p_{1}^{\prime} u_{1}^{\prime}= \begin{cases}u_{2}^{\prime} & \text { if } m \text { is odd }, \\
u_{2}^{\prime}+p_{3} u_{2}^{\prime} & \text { if } m \text { is even } ;\end{cases}\right.
$$$$
p_{2} u_{1}=p_{2} u_{1}^{\prime}=m p_{3} u_{0}, p_{2} u_{2}=p_{2} u_{2}^{\prime}=0 \text {; }
$$$$
p_{1} u_{2}= \begin{cases}u_{1}+m\left(\left[\frac{m}{2}\right] \cdot 1+\sum_{i=1}^{\left[\frac{m-2}{2}\right]}\left(\left[\frac{m}{2}\right]-i\right) q_{2 i}\right) u_{0} & \text { if } m \text { is odd } \\ u_{1}+p_{3} u_{1}+m\left(\sum_{i=1}^{\left[\frac{m-2}{2}\right]}\left(\left[\frac{m}{2}\right]-i\right) q_{2 i-1}\right) u_{0} & \text { if } m \text { is even }\end{cases}
$$$$
p_{1}^{\prime} u_{2}^{\prime}= \begin{cases}u_{1}^{\prime}+m\left(\left[\frac{m}{2}\right] \cdot 1+\sum_{i=1}^{\left[\frac{m-2}{2}\right]}\left(\left[\frac{m}{2}\right]-i\right) q_{2 i}\right) u_{0} \quad \text { if } m \text { is odd }, \\ u_{1}^{\prime}+p_{3} u_{1}^{\prime}+m\left(\sum_{i=1}^{\left[\frac{m-2}{2}\right]}\left(\left[\frac{m}{2}\right]-i\right) q_{2 i-1}\right) u_{0} \quad \text { if } m \text { is even; }\end{cases}
$$$$
p_{1}^{\prime} u_{2}=\left\{\begin{array}{l}
p_{3} u_{1}+m\left(\sum_{i=0}^{\left[\frac{m-2}{2}\right]}\left(\left[\frac{m}{2}\right]-i\right) q_{2 i+1}\right) u_{0} \quad \text { if } m \text { is odd }, \\
m\left(\left[\frac{m}{2}\right] \cdot 1+\sum_{i=1}^{\left[\frac{m-2}{2}\right]}\left(\left[\frac{m}{2}\right]-i\right) q_{2 i}\right) u_{0} \quad \text { if } m \text { is even } ;
\end{array}\right.
$$$$
p_{1} u_{2}^{\prime}= \begin{cases}p_{3} u_{1}^{\prime}+m\left(\sum_{i=0}^{\left[\frac{m-2}{2}\right]}\left(\left[\frac{m}{2}\right]-i\right) q_{2 i+1}\right) u_{0} & \text { if } m \text { is odd } \\ m\left(\left[\frac{m}{2}\right] \cdot 1+\sum_{i=1}^{\left[\frac{m-2}{2}\right]}\left(\left[\frac{m}{2}\right]-i\right) q_{2 i}\right) u_{0} & \text { if } m \text { is even }\end{cases}
$$

$p_{2} v=0,2 p_{1} w=2 p_{1}^{\prime} w=0$,

$u_{0} u_{2}=u_{0} u_{2}^{\prime}=0, u_{0}^{2}=\left(2-q_{2}\right) w, u_{0} u_{1}=u_{0} u_{1}^{\prime}=m q_{m-1} w$,

$u_{1} u_{1}^{\prime}= \begin{cases}m w & \text { if } m \text { is odd } \\ 0 & \text { if } m \text { is even }\end{cases}$ 


$$
\begin{aligned}
& u_{1} u_{2}^{\prime}=\left\{\begin{array}{ll}
p_{1}^{\prime} w & \text { if } m \text { is odd, } \\
0 & \text { if } m \text { is even; }
\end{array} \quad u_{1}^{\prime} u_{2}= \begin{cases}p_{1} w & \text { if } m \text { is odd }, \\
0 & \text { if } m \text { is even }\end{cases} \right. \\
& u_{2}^{2}-u_{1}^{2}=\left(u_{2}^{\prime}\right)^{2}-\left(u_{1}^{\prime}\right)^{2}= \begin{cases}m \sum_{i=1}^{\left[\frac{m-1}{2}\right]} q_{2 i} \cdot w & \text { if } m \text { is odd, } \\
m\left(1+p_{3}+\sum_{i=1}^{\left[\frac{m-1}{2}\right]} q_{2 i}\right) w & \text { if } m \text { is even; }\end{cases} \\
& u_{2} u_{2}^{\prime}= \begin{cases}m\left(p_{3}+\sum_{i=1}^{\left[\frac{m-1}{2}\right]} q_{2 i-1}\right) w & \text { if } m \text { is odd }, \\
m \sum_{i=0}^{\left[\frac{m-1}{2}\right]} q_{2 i+1} \cdot w & \text { if } m \text { is even; }\end{cases} \\
& u_{0} v=0, v^{2}=\left(u_{1}+u_{1}^{\prime}\right) w .
\end{aligned}
$$

Proof. Relations (4.2)-(4.17) are verified directly. For the remaining relations we use the translates of the corresponding elements that are described in Proposition 4.2. To illustrate the technique, we only verify relations (4.18). The remaining relations are established similarly, and we leave this to the reader. (Our choice of relations that we prove is partly explained by the fact that the description of the bases for $\operatorname{Im} \delta^{4}$ and $\operatorname{Im} \delta^{5}$ was not given, contrary to the description of those for the modules $\operatorname{Im} \delta^{i}$ with $i \leq 3$.)

A direct calculation, which uses the formulas for the translates $\mathrm{T}^{0}\left(u_{0}\right)$ and $\mathrm{T}^{2}(v)$ in Proposition 4.2, yields

$$
\mathrm{T}^{0}\left(u_{0}\right) \circ \mathrm{T}^{2}(v)=\left(-1 \otimes c+b c \otimes b,-c b \otimes c+1 \otimes b, \mathrm{O}_{3}\right) .
$$

Hence,

$$
\mu \circ \mathrm{T}^{0}\left(u_{0}\right) \circ \mathrm{T}^{2}(v)=\left(-c+b c b,-c b c+b, \mathrm{O}_{3}\right)=-\delta^{4}\left(b c, \mathrm{O}_{4}\right),
$$

and consequently, in $\operatorname{HH}^{*}(R)$ we have $u_{0} v=0$.

Now, using Remark 4.1. we easily obtain

$$
\mu \circ\left(\mathrm{T}^{0}\left(u_{1}\right)+\mathrm{T}^{0}\left(u_{1}^{\prime}\right)\right) \circ \mathrm{T}^{2}(w)=\left(0,1,1, \mathrm{O}_{4}\right) .
$$

On the other hand, Proposition 4.2 shows that

$$
\mathrm{T}^{0}(v) \circ \mathrm{T}^{3}(v)=\left(-\sum_{i=0}^{m-1}(b c)^{i} b \otimes c(b c)^{m-1-i}+\sum_{i=0}^{m-1}(b c)^{i} b \otimes c(b c)^{m-1-i},-1 \otimes 1,-1 \otimes 1, \mathrm{O}_{4}\right),
$$

whence

$$
\mu \mathrm{T}^{0}(v) \circ \mathrm{T}^{3}(v)=\left(0,-1,-1, \mathrm{O}_{4}\right) .
$$

Since $\delta^{5}\left(b, c, \mathrm{O}_{4}\right)=\left(0,2,2, \mathrm{O}_{4}\right)$, in $\mathrm{HH}^{*}(R)$ we have $v^{2}=\left(u_{1}+u_{1}^{\prime}\right) w$.

Proposition 4.6. The set $\mathcal{Y}\left(\right.$ see (4.1) ) generates $\mathrm{HH}^{*}(R)$ as a K-algebra.

Proof. Let $\mathcal{H}$ denote the $K$-subalgebra of $\mathrm{HH}^{*}(R)$ generated by the set $\mathcal{Y} \cup\{1\}$ (here 1 denotes the unity of the algebra $\left.\mathrm{HH}^{*}(R)\right)$. By Remark 4.4 we have $\mathrm{HH}^{0}(R) \subset \mathcal{H}$. The fact that the groups $\mathrm{HH}^{i}(R)$ with $2 \leq i \leq 5$ are contained in $\mathcal{H}$ is established with the help of the following formulas (see Propositions 3.11, 3.16, 3.21 and Corollary 3.26). 
Lemma 4.7. The following relations are valid:

a) for any $2 \leq i \leq m-1$, we have

$$
u_{0}^{(i)}= \begin{cases}\left(1+\sum_{t=1}^{\left[\frac{\left.\frac{m-1}{2}\right]}{2} q_{i+1-2 t}\right) u_{0}}\right. & \text { if } m \text { is odd, } \\ \sum_{t=0}^{\left[\frac{m-1}{2}\right]} q_{i-1-2 t} \cdot u_{0} & \text { if } m \text { is even; }\end{cases}
$$

b) if $m$ is even, then $v_{2}=p_{1} v, v_{2}^{\prime}=p_{1}^{\prime} v$; if $m$ is odd, then $\widetilde{v}_{2}=p_{1} v, \widetilde{v}_{2}^{\prime}=p_{1}^{\prime} v$; moreover, for any $m$ we have $v_{1}^{\prime}=p_{3} v$; $p_{1}^{\prime} w$

c) for any $2 \leq i \leq m-1$ we have $w_{0}^{(i)}=q_{i} w$; moreover, $w_{1}^{\prime}=p_{3} w, w_{2}=p_{1} w, w_{2}^{\prime}=$

$$
\begin{gathered}
w_{3}=\left\{\begin{array}{ll}
u_{1}^{2} & \text { if } m \text { is odd }, \\
u_{1}^{2}-m w & \text { if } m \text { is even } ; \\
u_{1} u_{2} & \text { if } m \text { is odd }, \\
u_{1} u_{2}-p_{1} w & \text { if } m \text { is even } ;
\end{array} \quad w_{3}^{\prime}= \begin{cases}\left(u_{1}^{\prime}\right)^{2} & \text { if } m \text { is odd }, \\
\left(u_{1}^{\prime}\right)^{2}-m w & \text { if } m \text { is even } ; \\
u_{1}^{\prime} u_{2}^{\prime} & \text { if } m \text { is odd }, \\
u_{1}^{\prime} u_{2}^{\prime}-p_{1}^{\prime} w & \text { if } m \text { is even } ;\end{cases} \right. \\
w_{5}=p_{3} w_{4}, w_{6}=p_{3} w_{3}, w_{5}^{\prime}=p_{3} w_{4}^{\prime}, w_{6}^{\prime}=p_{3} w_{3}^{\prime} ;
\end{gathered}
$$

d)

$$
\begin{aligned}
& t_{1}=u_{2} v, t_{2}=u_{1} v, t_{3}=p_{3} u_{1} v, t_{4}=p_{3} u_{2} v, \\
& t_{1}^{\prime}=u_{2}^{\prime} v, t_{2}^{\prime}=u_{1}^{\prime} v, t_{3}^{\prime}=p_{3} u_{1}^{\prime} v, t_{4}^{\prime}=p_{3} u_{2}^{\prime} v .
\end{aligned}
$$

Proof. These relations are verified directly, as in the proof of Proposition 4.5 .

We also need the following auxiliary statement.

Lemma 4.8. Set $\bar{u}:=u_{1}+u_{1}^{\prime} \in \mathrm{HH}^{2}(R)$. For any $r \geq 3$, the translates $\mathrm{T}^{r}(\bar{u})$ can be taken so that their matrices have the following block form:

$$
\mathrm{T}^{r}(\bar{u})=\left(\begin{array}{cc}
\star & \mathrm{O} \\
\star & \operatorname{id}_{\Lambda^{4}}
\end{array}\right),
$$

where $\mathrm{id}_{\Lambda^{4}}$ is the matrix of the identity map of the module $\Lambda^{4}$.

Proof. By Proposition 4.5, we have

$$
\mathrm{T}^{2}(\bar{u})=\left(\begin{array}{ccccc}
0 & 1 & 1 & 0 & 0 \\
\star & 0 & 0 & 1 & 0 \\
\star & 0 & 0 & 0 & 1
\end{array}\right) .
$$

Hence, $\mathrm{T}^{2}(\bar{u}) d_{4}^{Q}=\left(\star d_{2}^{Q}\right)$. Since we need the identity $\mathrm{T}^{2}(\bar{u}) d_{4}^{Q}=d_{2}^{Q} \mathrm{~T}^{3}(\bar{u})$, we can choose the translate $\mathrm{T}^{3}(\bar{u})$ with a matrix of the desired form. With the help of similar arguments, we complete the proof by induction on $r$.

We continue the proof of Proposition 4.6. Using induction on $n$, we check that $\mathrm{HH}^{n}(R) \subset \mathcal{H}$. Assume that $n \geq 6$, and put

$$
f=\left(f^{\prime}, f^{\prime \prime}\right): Q_{n}=Q_{n-4} \oplus X_{n} \rightarrow R
$$

with $f^{\prime} \in \operatorname{Hom}_{\Lambda}\left(Q_{n-4}, R\right)$ and $f^{\prime \prime} \in \operatorname{Hom}_{\Lambda}\left(X_{n}, R\right)$. Observe that $f^{\prime \prime} \in \operatorname{Ker} \delta_{\mathcal{X}}^{n}$.

a) Assume additionally that, in (4.19), we have $f^{\prime \prime}=0$. Clearly, also $f^{\prime} \in \operatorname{Ker} \delta^{n-4}$. Furthermore, by Remark 4.1 we have $f=f^{\prime} \circ \pi_{n}=f^{\prime} \circ \mathrm{T}^{n-4}(w)$. Consequently, the relation $f=f^{\prime} w$ is fulfilled in $\operatorname{HH}^{*}(R)$, and it remains to apply the induction hypothesis to $f^{\prime}$. 
b) Now we consider the case where $n$ is even: $n=2 l$. Using the form of the matrix of the differential $d_{2 l}^{Q}$ (see (3.81)) and Lemmas 3.25 and 3.10, we see that $g:=\left(\mathrm{O}_{n-5}, f^{\prime \prime}\right)$ lies in $\operatorname{Ker} \delta^{n-2}$. By Lemma 4.8, it follows that $g \circ \mathrm{T}^{n-2}(\bar{u})=\left(\star, f^{\prime \prime}\right)$. Therefore, $f_{1}:=f-g \bar{u}=\left(\star, \mathrm{O}_{4}\right)$, and it remains to apply part a) of the proof to $f_{1}$ and the induction hypothesis to $g$.

c) Now we assume that $n$ is odd: $n=2 l+1$. Set $f^{\prime \prime}=\left(r_{1}, r_{2}, r_{3}, r_{4}\right)$ with $r_{i} \in R$. Since $B^{+} * r_{3}=0=C^{+} * r_{4}$, by Lemma 3.15 we have $r_{3}=B^{-} * u, r_{4}=C^{-} * u^{\prime}$ for some $u, u^{\prime} \in R$. Replacing $f$ by the element $f-\delta^{2 l}\left(\mathrm{O}_{2 l-1}, u, u^{\prime}\right)$, we may assume that $r_{3}=0=r_{4}$ for the initial $f$. From the relation $\delta_{\mathcal{X}}^{2 l+1}\left(f^{\prime \prime}\right)=0$ it follows that $B^{-} * r_{1}=0$ and $C^{-} * r_{2}=0$. It is easily seen that $g:=\left(\mathrm{O}_{2 l-6},-r_{1}(b c)^{m}, r_{2}(b c)^{m}, r_{1}, r_{2}, 0,0\right) \in \operatorname{Hom}_{\Lambda}\left(Q_{2 l-1}, R\right)$ is a cocycle. Hence, $f_{1}:=f-g \bar{u}=\left(\star, \mathrm{O}_{4}\right)$, and the desired statement is established by an argument similar to part b) of the proof.

Corollary 4.9. If $R=\mathbb{Z}\left[D_{4 m}\right]$, then the algebra $\mathrm{HH}^{*}(R)$ is commutative.

Proof. This follows immediately from Proposition 4.6 and from the relations satisfied by the elements of the set $\mathcal{Y}$ (see Proposition 4.5), because there is a unique generator with odd degree, namely $v$, and for this generator we have $2 v=0$.

Let $\mathcal{A}=\mathcal{A}_{m}=\mathbb{Z}[\mathcal{X}] / I_{m}$ be a graded $\mathbb{Z}$-algebra defined in $\S 1$, where $\mathcal{X}$ is as in (1.5), and $I_{m}$ is the corresponding ideal of relations (see (1.6)-(1.26)). For an element $x \in \mathbb{Z}[\mathcal{X}]$, we keep the notation $x$ also for its image in $\mathcal{A}$ under the canonical map $\mathbb{Z}[\mathcal{X}] \rightarrow \mathcal{A}$. The nonzero images of monomials in $\mathbb{Z}[\mathcal{X}]$ under this map are also called monomials. Any element $x \in \mathcal{A}$ is represented as a linear combination of monomials (with coefficients in $\mathbb{Z}$ ). Propositions 4.5 and 4.6 imply that there exists a surjective homomorphism $\varphi: \mathcal{A} \rightarrow \mathrm{HH}^{*}(R)$ of graded $\mathbb{Z}$-algebras that takes the generators belonging to $\mathcal{X}$ to the corresponding generators in $\mathcal{Y}$ (see (4.1)). Note that no ambiguity is caused by employing the same letter to denote elements of both sets that correspond to each other. Let $\mathcal{A}=\bigoplus_{n \geq 0} \mathcal{A}^{n}$ be the direct decomposition of the algebra $\mathcal{A}$ into homogeneous direct summands, and let $\varphi_{n}: \mathcal{A}^{n} \rightarrow \mathrm{HH}^{n}(R)$ be the restriction of $\varphi$ to the corresponding homogeneous component.

Now, Theorem 1.1 is a consequence of the following statement.

Proposition 4.10. For any $n \geq 0, \varphi_{n}: \mathcal{A}^{n} \rightarrow \operatorname{HH}^{n}(R)$ is an isomorphism.

Proof. By definition, reduction of a monomial $a$ in $\mathcal{A}$ is the process of replacement of some submonomials of $a$ by other elements of $\mathcal{A}$ in accordance with the following rules ( $x \mapsto y$ means the replacement of every occurrence of the monomial $x$ by the element $y$ ):

$$
\begin{aligned}
& p_{1}^{2} \mapsto \begin{cases}m\left(1+\sum_{i=1}^{\left[\frac{m-1}{2}\right]} q_{2 i}\right) & \text { if } m \text { is odd, } \\
m\left(1+p_{3}+\sum_{i=1}^{\left[\frac{m-1}{2}\right]} q_{2 i}\right) & \text { if } m \text { is even; }\end{cases} \\
& \left(p_{1}^{\prime}\right)^{2} \mapsto \begin{cases}m\left(1+\sum_{i=1}^{\left[\frac{m-1}{2}\right]} q_{2 i}\right) & \text { if } m \text { is odd } \\
m\left(1+p_{3}+\sum_{i=1}^{\left[\frac{m-1}{2}\right]} q_{2 i}\right) & \text { if } m \text { is even; }\end{cases}
\end{aligned}
$$


$p_{1} p_{1}^{\prime} \mapsto \begin{cases}m\left(p_{3}+\sum_{i=1}^{\left[\frac{m}{2}\right]} q_{2 i-1}\right) & \text { if } m \text { is odd, } \\ m \sum_{i=1}^{\left[\frac{m}{2}\right]} q_{2 i-1} & \text { if } m \text { is even; }\end{cases}$

$p_{1} p_{3} \mapsto\left\{\begin{array}{ll}p_{1}^{\prime} & \text { if } m \text { is odd, } \\ p_{1} & \text { if } m \text { is even; }\end{array} \quad p_{1}^{\prime} p_{3} \mapsto \begin{cases}p_{1} & \text { if } m \text { is odd, } \\ p_{1}^{\prime} & \text { if } m \text { is even; }\end{cases}\right.$

$p_{1} p_{2} \mapsto 2 p_{1}^{\prime}, \quad p_{1}^{\prime} p_{2} \mapsto 2 p_{1}, p_{3}^{2} \mapsto 1 ;$

$p_{2} p_{3} \mapsto q_{m-1}, p_{2}^{m} \mapsto 2 p_{3}-g_{m}\left(p_{2}\right)$;

$p_{2}^{m-1} u_{0} \mapsto \begin{cases}-\left(1+g_{m-1}\left(p_{2}\right)+\sum_{i=1}^{\left[\frac{m-2}{2}\right]} q_{m-1-2 i}\right) u_{0} & \text { if } m \text { is odd, } \\ -\left(g_{m-1}\left(p_{2}\right)+\sum_{i=1}^{\left[\frac{m-2}{2}\right]} q_{m-1-2 i}\right) u_{0} & \text { if } m \text { is even; }\end{cases}$

$p_{3} u_{0} \mapsto \begin{cases}-\sum_{i=0}^{\left[\frac{m-3}{2}\right]} q_{m-2-2 i} \cdot u_{0} & \text { if } m \text { is odd, } \\ -\left(1+\sum_{i=0}^{\left[\frac{m-3}{2}\right]} q_{m-2-2 i}\right) u_{0} & \text { if } m \text { is even; }\end{cases}$

$p_{1} u_{1} \mapsto \begin{cases}u_{2} & \text { if } m \text { is odd, } \\ u_{2}+p_{3} u_{2} & \text { if } m \text { is even; }\end{cases}$

$p_{1}^{\prime} u_{1}^{\prime} \mapsto \begin{cases}u_{2}^{\prime} & \text { if } m \text { is odd, } \\ u_{2}^{\prime}+p_{3} u_{2}^{\prime} & \text { if } m \text { is even; }\end{cases}$

$p_{1} u_{1}^{\prime} \mapsto p_{3} u_{2}^{\prime}$ if $m$ is odd; $p_{1}^{\prime} u_{1} \mapsto p_{3} u_{2}$ if $m$ is odd;

$p_{2} u_{1} \mapsto m p_{3} u_{0}, \quad p_{2} u_{1}^{\prime} \mapsto m p_{3} u_{0} ;$

$p_{1} u_{2} \mapsto \begin{cases}u_{1}+m\left(\left[\frac{m}{2}\right] \cdot 1+\sum_{i=1}^{\left[\frac{m-2}{2}\right]}\left(\left[\frac{m}{2}\right]-i\right) q_{2 i}\right) u_{0} & \text { if } m \text { is odd } \\ u_{1}+p_{3} u_{1}+m\left(\sum_{i=1}^{\left[\frac{m-2}{2}\right]}\left(\left[\frac{m}{2}\right]-i\right) q_{2 i-1}\right) u_{0} & \text { if } m \text { is even }\end{cases}$

$p_{1}^{\prime} u_{2}^{\prime} \mapsto \begin{cases}u_{1}^{\prime}+m\left(\left[\frac{m}{2}\right] \cdot 1+\sum_{i=1}^{\left[\frac{m-2}{2}\right]}\left(\left[\frac{m}{2}\right]-i\right) q_{2 i}\right) u_{0} & \text { if } m \text { is odd } \\ u_{1}^{\prime}+p_{3} u_{1}^{\prime}+m\left(\sum_{i=1}^{\left[\frac{m-2}{2}\right]}\left(\left[\frac{m}{2}\right]-i\right) q_{2 i-1}\right) u_{0} & \text { if } m \text { is even }\end{cases}$

$p_{1}^{\prime} u_{2} \mapsto \begin{cases}p_{3} u_{1}+m\left(\sum_{i=0}^{\left[\frac{m-2}{2}\right]}\left(\left[\frac{m}{2}\right]-i\right) q_{2 i+1}\right) u_{0} & \text { if } m \text { is odd, } \\ m\left(\left[\frac{m}{2}\right] \cdot 1+\sum_{i=1}^{\left[\frac{m-2}{2}\right]}\left(\left[\frac{m}{2}\right]-i\right) q_{2 i}\right) u_{0} & \text { if } m \text { is even; }\end{cases}$

$p_{1} u_{2}^{\prime} \mapsto \begin{cases}p_{3} u_{1}^{\prime}+m\left(\sum_{i=0}^{\left[\frac{m-2}{2}\right]}\left(\left[\frac{m}{2}\right]-i\right) q_{2 i+1}\right) u_{0} & \text { if } m \text { is odd, } \\ m\left(\left[\frac{m}{2}\right] \cdot 1+\sum_{i=1}^{\left[\frac{m-2}{2}\right]}\left(\left[\frac{m}{2}\right]-i\right) q_{2 i}\right) u_{0} & \text { if } m \text { is even; }\end{cases}$ 


$$
\begin{aligned}
& u_{0}^{2} \mapsto\left(2-q_{2}\right) w, \quad u_{0} u_{1} \mapsto m q_{m-1} w, \quad u_{0} u_{1}^{\prime} \mapsto m q_{m-1} w ; \\
& u_{1} u_{1}^{\prime} \mapsto m w \text { if } m \text { is odd; } \\
& u_{1} u_{2}^{\prime} \mapsto p_{1}^{\prime} w \text { if } m \text { is odd; } \\
& u_{1}^{\prime} u_{2} \mapsto p_{1} w \text { if } m \text { is odd; } \\
& u_{1}^{2} \mapsto \begin{cases}u_{2}^{2}+m \sum_{i=1}^{\left[\frac{m-1}{2}\right]} q_{2 i} \cdot w & \text { if } m \text { is odd }, \\
u_{2}^{2}+m\left(1+p_{3}+\sum_{i=1}^{\left[\frac{m-1}{2}\right]} q_{2 i}\right) w & \text { if } m \text { is even; }\end{cases} \\
& \left(u_{1}^{\prime}\right)^{2} \mapsto \begin{cases}\left(u_{2}^{\prime}\right)^{2}+m \sum_{i=1}^{\left[\frac{m-1}{2}\right]} q_{2 i} \cdot w & \text { if } m \text { is odd, } \\
\left(u_{2}^{\prime}\right)^{2}+m\left(1+p_{3}+\sum_{i=1}^{\left[\frac{m-1}{2}\right]} q_{2 i}\right) w & \text { if } m \text { is even; }\end{cases} \\
& u_{2} u_{2}^{\prime} \mapsto \begin{cases}m\left(p_{3}+\sum_{i=1}^{\left[\frac{m-1}{2}\right]} q_{2 i-1}\right) w & \text { if } m \text { is odd, } \\
m \sum_{i=0}^{\left[\frac{m-1}{2}\right]} q_{2 i+1} \cdot w & \text { if } m \text { is even; }\end{cases} \\
& v^{2} \mapsto\left(u_{1}+u_{1}^{\prime}\right) w .
\end{aligned}
$$

Any replacement on the above list is called an elementary step of reduction.

On the ring $\mathbb{Z}[\mathcal{X}]$, we introduce a new grading with the help of the function $\chi: \mathbb{Z}[\mathcal{X}] \rightarrow$ $\mathbb{N}_{0}$ such that, for any elementary step of reduction $x \mapsto y$, we have $\chi(x)>\chi(y)$ (more precisely, here $x$ and $y$ are the corresponding elements of $\mathbb{Z}[\mathcal{X}]$ ). For example, it is easily seen that the degree function has such properties if we introduce a new grading $\chi$ on $\mathbb{Z}[\mathcal{X}]$ as follows:

$$
\begin{gathered}
\chi\left(p_{1}\right)=\chi\left(p_{1}^{\prime}\right)=2 m-1, \chi\left(p_{2}\right)=1, \chi\left(p_{3}\right)=m-1, \\
\chi\left(u_{0}\right)=2, \chi\left(u_{1}\right)=\chi\left(u_{1}^{\prime}\right)=\chi(v)=m+1, \\
\chi\left(u_{2}\right)=\chi\left(u_{2}^{\prime}\right)=m, \chi(w)=1 .
\end{gathered}
$$

Since the values of $\chi$ strictly decrease under reduction, after finitely many steps we obtain monomials or linear combinations of monomials that do not admit any elementary step of reduction. We say that a presentation of an element $f \in \mathcal{A}$ as a linear combination of monomials has normal form if reduction cannot be applied to any of these monomials. Since these elementary steps of reduction correspond to some relations in the algebra $\mathcal{A}$ (see (1.6)-(1.26)), any element $f \in \mathcal{A}$ admits at least one presentation in the normal form.

1) First, assume that $n=0$. Let

$$
f=p_{1}^{\alpha_{1}}\left(p_{1}^{\prime}\right)^{\alpha_{1}^{\prime}} p_{2}^{\alpha_{2}} p_{3}^{\alpha_{3}} \in \mathcal{A}^{0}
$$

be a monomial in the normal form. By the steps of reduction in (4.20)-4.25), we have $\alpha_{1}+\alpha_{1}^{\prime}+\alpha_{3} \in\{0,1\}$ and $0 \leq \alpha_{2} \leq m-1$. Hence, $f$ coincides with one of the monomials

$$
p_{1}, p_{1}^{\prime}, p_{3}, p_{2}^{i} \quad(0 \leq i \leq m-1) .
$$

Consequently, the Abelian group $\mathcal{A}^{0}$ is generated by the set consisting of $m+3$ elements and simultaneously admits an epimorphism onto the free Abelian group $\mathrm{HH}^{0}(R)$ of rank $m+3$. This immediately implies that $\varphi: \mathcal{A}^{0} \rightarrow \mathrm{HH}^{0}(R)$ is an isomorphism.

2) Assume that $n \geq 1$. We shall prove that the cardinality $\left|\mathcal{A}^{n}\right|$ does not exceed the cardinality $\left|\mathrm{HH}^{n}(R)\right|$, which implies that $\varphi_{n}: \mathcal{A}^{n} \rightarrow \mathrm{HH}^{n}(R)$ is an isomorphism. 
Let $f \in \mathcal{A}^{n}$ be a monomial in the normal form. Put $f=f_{0} f^{\prime}$, where $f_{0} \in \mathcal{A}^{0}$ and

$$
f^{\prime}=u_{0}^{\beta_{0}} u_{1}^{\beta_{1}} u_{2}^{\beta_{2}}\left(u_{1}^{\prime}\right)^{\gamma_{1}}\left(u_{2}^{\prime}\right)^{\gamma_{2}} v^{\varepsilon} w^{l} .
$$

Using the list of elementary steps of reduction, we see that $\beta_{0}, \varepsilon \in\{0,1\}, \beta_{1}+\gamma_{1} \leq 1$ and $\beta_{2} \gamma_{2}=0$. Next, we consider several cases successively.

a) Assume additionally that $\beta_{0}=1$. Then $f^{\prime}$ does not involve any of the following elements: $u_{1}$ and $u_{1}^{\prime}$ (because the products $u_{0} u_{1}$ and $u_{0} u_{1}^{\prime}$ can be reduced), $u_{2}, u_{2}^{\prime}$, and $v$ (because $\left.u_{0} u_{2}=u_{0} u_{2}^{\prime}=u_{0} v=0\right), p_{1}, p_{1}^{\prime}$, and $p_{3}$ (because $p_{3} u_{0}$ can be reduced and $p_{1} u_{0}=p_{1}^{\prime} u_{0}=0$ ). Moreover, in (4.26) we have $\alpha_{2} \leq m-2$ (because $p_{2}^{m-1} u_{0}$ can be reduced). Consequently, $f$ coincides with one of the monomials

$$
p_{2}^{i} u_{0} w^{l} \quad\left(0 \leq i \leq m-2, l \in \mathbb{N}_{0}\right) .
$$

b) Assume that $f^{\prime}$ involves $u_{1}$. Then $f^{\prime}$ cannot involve $u_{1}^{\prime}$ and $u_{2}^{\prime}$ (because $u_{1} u_{1}^{\prime}$ and $u_{1} u_{2}^{\prime}$ are zero if $m$ is even, or can be reduced if $m$ is odd). Moreover, $f_{0}$ cannot involve $p_{1}, p_{1}^{\prime}, p_{2}$ (because $p_{1} u_{1}, p_{2} u_{1}$ can be reduced, and $p_{1}^{\prime} u_{1}$ either can be reduced or is equal to zero). Consequently, $f$ coincides with one of the monomials

$$
u_{1} u_{2}^{t} w^{l}, u_{1} u_{2}^{t} v w^{l}, p_{3} u_{1} u_{2}^{t} w^{l}, p_{3} u_{1} u_{2}^{t} v w^{l} \quad\left(t, l \in \mathbb{N}_{0}\right) .
$$

c) If $f^{\prime}$ involves $u_{1}^{\prime}$, then, by symmetry with case b), we see that $f$ coincides with one of the following monomials:

$$
u_{1}^{\prime}\left(u_{2}^{\prime}\right)^{t} w^{l}, u_{1}^{\prime}\left(u_{2}^{\prime}\right)^{t} v w^{l}, p_{3} u_{1} \prime\left(u_{2}^{\prime}\right)^{t} w^{l}, p_{3} u_{1}^{\prime}\left(u_{2}^{\prime}\right)^{t} v w^{l} \quad\left(t, l \in \mathbb{N}_{0}\right) .
$$

d) Assume that $u_{0}, u_{1}, u_{1}^{\prime}$ do not occur in $f^{\prime}$ and $u_{2}$ occurs in $f^{\prime}$. As in case b), we prove that $f$ has the form $f=p_{3}^{\alpha_{3}} u_{2}^{\beta_{2}} v^{\varepsilon} w^{l}$. Consequently, $f$ coincides with one of the following monomials:

$$
u_{2}^{t} w^{l}, u_{2}^{t} v w^{l}, p_{3} u_{2}^{t} w^{l}, p_{3} u_{2}^{t} v w^{l} \quad\left(t \in \mathbb{N}, l \in \mathbb{N}_{0}\right) .
$$

e) If $u_{0}, u_{1}, u_{1}^{\prime}, u_{2}$ do not occur in $f^{\prime}$ and $u_{2}^{\prime}$ occurs in $f^{\prime}$, then, by symmetry with case d), we see that $f$ coincides with one of the following monomials:

$$
\left(u_{2}^{\prime}\right)^{t} w^{l},\left(u_{2}^{\prime}\right)^{t} v w^{l}, p_{3}\left(u_{2}^{\prime}\right)^{t} w^{l}, p_{3}\left(u_{2}^{\prime}\right)^{t} v w^{l} \quad\left(t \in \mathbb{N}, l \in \mathbb{N}_{0}\right) .
$$

f) Assume that $u_{0}, u_{1}, u_{1}^{\prime}, u_{2}, u_{2}^{\prime}$ do not occur in $f^{\prime}$ and $v$ occurs in $f^{\prime}$. Then $f_{0}$ does not involve $p_{2}$ (because $p_{2} v=0$ ). Consequently, $f$ coincides with one of the following monomials:

$$
v w^{l}, p_{1} v w^{l}, p_{1}^{\prime} v w^{l}, p_{3} v w^{l} .
$$

g) Finally, if $u_{0}, u_{1}, u_{1}^{\prime}, u_{2}, u_{2}^{\prime}, v$ do not occur in $f^{\prime}$, then, clearly, $f$ coincides with one of the monomials

$$
w^{l}, p_{1} w^{l}, p_{1}^{\prime} w^{l}, p_{3} w^{l}, p_{2}^{i} w^{l} \quad(l \in \mathbb{N}, 0 \leq i \leq m-1) .
$$

Now, considering the degrees of the elements in (4.27)-(4.33) (with respect to the initial grading on the algebra $\mathcal{A}$ ), we form Table 1

The defining relations of the algebra $\mathcal{A}$ show that the monomials $p_{2}^{i} w^{k}(0 \leq i \leq m-1)$, $p_{2}^{i} u_{0} w^{k}(0 \leq i \leq m-2)$, and $p_{3} w^{k}$ admit scalar coefficients modulo $2 m$, and the remaining monomials on the above list admit coefficients modulo 2 . The listed monomials of degree $4 k+r$ with $r \in\{0,1,2,3\}$ generate the group $\mathcal{A}^{4 k+r}$. From this, it is easily seen that for $k \geq 1$ we have $\left|\mathcal{A}^{4 k}\right| \leq(2 m)^{m+1} 2^{8 k+2}$, and for $k \geq 0$ we have $\left|\mathcal{A}^{4 k+1}\right| \leq 2^{8 k \text {, }}$ $\left|\mathcal{A}^{4 k+2}\right| \leq(2 m)^{m-1} 2^{8(k+1)},\left|\mathcal{A}^{4 k+3}\right| \leq 2^{8 k+4}$. This completes the proof of the inequality $\left|\mathcal{A}^{n}\right| \leq\left|\mathrm{HH}^{n}(R)\right|$ for all $n \geq 1$. 
TABLE 1

\begin{tabular}{|c|c|}
\hline Degree & Normal forms \\
\hline \multirow{4}{*}{$4 k, k>0$} & $p_{3}^{\alpha} u_{1}^{\beta} u_{2}^{2(k-l)-1} w^{l}(0 \leq l \leq k-1 ; \alpha, \beta \in\{0,1\})$, \\
& $p_{3}^{\alpha}\left(u_{1}^{\prime}\right)^{\beta}\left(u_{2}^{\prime}\right)^{2(k-l)-1} w^{l}(0 \leq l \leq k-1 ; \alpha, \beta \in\{0,1\})$, \\
& $p_{2}^{i} w^{k}(0 \leq i \leq m-1), p_{1} w^{k}, p_{1}^{\prime} w^{k}, p_{3} w^{k}$ \\
\hline \multirow{4}{*}{$4 k+1$} & $p_{3}^{\alpha} u_{1} u_{2}^{2(k-l-1)} v w^{l}(0 \leq l \leq k-1 ; \alpha \in\{0,1\})$, \\
& $p_{3}^{\alpha} u_{2}^{2(k-l)-1)} v w^{l}(0 \leq l \leq k-1 ; \alpha \in\{0,1\})$, \\
& $p_{3}^{\alpha} u_{1}^{\prime}\left(u_{2}^{\prime}\right)^{2(k-l-1)} v w^{l}(0 \leq l \leq k-1 ; \alpha \in\{0,1\})$, \\
& $p_{3}^{\alpha}\left(u_{2}^{\prime}\right)^{2(k-l)-1)} v w^{l}(0 \leq l \leq k-1 ; \alpha \in\{0,1\})$, \\
\hline \multirow{4}{*}{$4 k+2$} & $p_{3}^{\alpha} u_{1}^{\beta} u_{2}^{2(k-l)} w^{l}(0 \leq l \leq k, \alpha, \beta \in\{0,1\})$, \\
& $p_{3}^{\alpha}\left(u_{1}^{\prime}\right)^{\beta}\left(u_{2}^{\prime}\right)^{2(k-l)-1} w^{l}(0 \leq l \leq k, \alpha, \beta \in\{0,1\})$, \\
& $p_{2}^{i} u_{0} w^{k}(0 \leq i \leq m-2)$ \\
\hline \multirow{4}{*}{$4 k+3$} & $p_{3}^{\alpha} u_{1} u_{2}^{2(k-l)-1} w^{l}(0 \leq l \leq k-1 ; \alpha \in\{0,1\})$, \\
& $p_{3}^{\alpha} u_{1}^{\prime}\left(u_{2}^{\prime}\right)^{2(k-l)-1} w^{l}(0 \leq l \leq k-1 ; \alpha \in\{0,1\})$, \\
& $p_{3}^{\alpha} u_{2}^{2(k-l)} w^{l}(0 \leq l \leq k ; \alpha \in\{0,1\})$, \\
& $p_{3}^{\alpha}\left(u_{2}^{\prime}\right)^{2(k-l)} w^{l}(0 \leq l \leq k-1 ; \alpha \in\{0,1\})$, \\
$p_{1} v w^{k}, p_{1}^{\prime} v w^{k}$ \\
\hline
\end{tabular}

Corollary 4.11. If $m$ is odd, then the cohomology algebra $\mathrm{HH}^{*}\left(\mathbb{Z}\left[D_{4 m}\right]\right)$ is generated by the homogeneous elements

$$
p_{1}, p_{2}, p_{3}, u_{0}, u_{1}, u_{1}^{\prime}, v, w
$$

of degrees

$$
\operatorname{deg} p_{i}=0(i=1,2,3), \operatorname{deg} u_{0}=\operatorname{deg} u_{1}=\operatorname{deg} u_{1}^{\prime}=2, \operatorname{deg} v=3, \operatorname{deg} w=4,
$$

and these elements satisfy the following defining relations:

- of degree 0 :

$$
\begin{aligned}
& p_{1}^{2}=m\left(1+\sum_{i=1}^{\left[\frac{m-1}{2}\right]} q_{2 i}\right), \\
& p_{1} p_{2}=2 p_{1} p_{3}, \quad q_{m}=2 p_{3}, \quad p_{2} p_{3}=q_{m-1}, \quad p_{3}^{2}=1 ;
\end{aligned}
$$

- of degree 2 :

$$
\begin{aligned}
& p_{1} u_{0}=2 m u_{0}=2 u_{1}=2 u_{1}^{\prime}=0, \\
& \left(1+\sum_{i=0}^{\left[\frac{m-2}{2}\right]} q_{m-1-2 i}\right) u_{0}=0 \\
& p_{3} u_{0}=-\left(\sum_{i=0}^{\left[\frac{m-3}{2}\right]} q_{m-2-2 i}\right) u_{0}, \\
& p_{2} u_{1}=p_{2} u_{1}^{\prime}=m p_{3} u_{0} ;
\end{aligned}
$$

- of degree 3 :

$$
2 v=p_{2} v=0
$$


- of degree 4:

$$
\begin{aligned}
& 2 m w=2 p_{1} w=0, u_{0}^{2}-\left(2-q_{2}\right) w \\
& u_{0} u_{1}=u_{0} u_{1}=m q_{m-1} w, u_{1} u_{1}^{\prime}=m w ;
\end{aligned}
$$

- of degree 5 :

$$
u_{0} v=0
$$

- of degree 6 :

$$
v^{2}=\left(u_{1}+u_{1}^{\prime}\right) w .
$$

Proof. We can exclude the elements $p_{1}^{\prime}, u_{2}, u_{2}^{\prime}$ from the set $\mathcal{X}$ (see (1.5)), by using the generators (1.8) and (1.14) of the ideal $I_{m}$ of relations. It can be checked that the relations that involve excluded elements follow from relations (4.34)-(4.44).

Corollary 4.12. If $m$ is even, then the cohomology algebra $\mathrm{HH}^{*}\left(\mathbb{Z}\left[D_{4 m}\right]\right)$ is generated by the homogeneous elements

$$
p_{1}, p_{1}^{\prime}, p_{2}, p_{3}, u_{0}, u_{1}, u_{1}^{\prime}, u_{2}, u_{2}^{\prime}, v, w
$$

of degrees

$$
\begin{gathered}
\operatorname{deg} p_{1}^{\prime}=\operatorname{deg} p_{i}=0 \quad(i=1,2,3) ; \\
\operatorname{deg} u_{0}=\operatorname{deg} u_{i}=\operatorname{deg} u_{i}^{\prime}=2 \text { for } i=1,2 ; \quad \operatorname{deg} v=3, \operatorname{deg} w=4,
\end{gathered}
$$

and these elements satisfy the following defining relations:

— of degree 0:

$$
\begin{aligned}
& p_{1}^{2}=\left(p_{1}^{\prime}\right)^{2}=m\left(1+p_{3}+\sum_{i=1}^{\left[\frac{m-1}{2}\right]} q_{2 i}\right), \\
& p_{1} p_{1}^{\prime}=m \sum_{i=1}^{\left[\frac{m}{2}\right]} q_{2 i-1}, \\
& p_{1} p_{3}=p_{1}, p_{1}^{\prime} p_{3}=p_{1}^{\prime}, \\
& p_{1} p_{2}=2 p_{1}^{\prime}, p_{1}^{\prime} p_{2}=2 p_{1}, q_{m}=2 p_{3}, p_{2} p_{3}=q_{m-1}, p_{3}^{2}=1 ;
\end{aligned}
$$

- of degree 2:

$$
\begin{aligned}
& p_{1} u_{0}=p_{1}^{\prime} u_{0}=2 m u_{0}=2 u_{i}=2 u_{i}^{\prime}=0 \text { for } i \in\{1,2\} ; \\
& \sum_{i=0}^{\left[\frac{m-2}{2}\right]} q_{m-1-2 i} \cdot u_{0}=0, \\
& p_{3} u_{0}=-\left(1+\sum_{i=0}^{\left[\frac{m-3}{2}\right]} q_{m-2-2 i}\right) u_{0}, \\
& p_{1} u_{1}=u_{2}+p_{3} u_{2}, p_{1}^{\prime} u_{1}^{\prime}=u_{2}^{\prime}+p_{3} u_{2}^{\prime}, \\
& p_{1}^{\prime} u_{1}=p_{1} u_{1}^{\prime}=p_{2} u_{2}=p_{2} u_{2}^{\prime}=0, \\
& p_{2} u_{1}=p_{2} u_{1}^{\prime}=m p_{3} u_{0}, \\
& p_{1} u_{2}=u_{1}+p_{3} u_{1}+m\left(\sum_{i=1}^{\left[\frac{m-2}{2}\right]}\left(\left[\frac{m}{2}\right]-i\right) q_{2 i-1}\right) u_{0},
\end{aligned}
$$




$$
\begin{aligned}
& p_{1}^{\prime} u_{2}^{\prime}=u_{1}^{\prime}+p_{3} u_{1}^{\prime}+m\left(\sum_{i=1}^{\left[\frac{\left.\frac{m-2}{2}\right]}{2}\right.}\left(\left[\frac{m}{2}\right]-i\right) q_{2 i-1}\right) u_{0}, \\
& p_{1}^{\prime} u_{2}=m\left(\left[\frac{m}{2}\right] \cdot 1+\sum_{i=1}^{\left[\frac{m-2}{2}\right]}\left(\left[\frac{m}{2}\right]-i\right) q_{2 i}\right) u_{0}, \\
& p_{1} u_{2}^{\prime}=m\left(\left[\frac{m}{2}\right] \cdot 1+\sum_{i=1}^{\left[\frac{m-2}{2}\right]}\left(\left[\frac{m}{2}\right]-i\right) q_{2 i}\right) u_{0}
\end{aligned}
$$

- of degree 3 :

$$
2 v=p_{2} v=0
$$

- of degree 4:

$$
\begin{aligned}
& 2 m w=2 p_{1} w=2 p_{1}^{\prime} w=u_{0} u_{2}=u_{0} u_{2}^{\prime}=0, \\
& u_{1} u_{1}^{\prime}=u_{1} u_{2}^{\prime}=u_{1}^{\prime} u_{2}=0, \\
& u_{0}^{2}-\left(2-q_{2}\right) w, u_{0} u_{1}=u_{0} u_{1}^{\prime}=m q_{m-1} w, \\
& u_{2}^{2}-u_{1}^{2}=\left(u_{2}^{\prime}\right)^{2}-\left(u_{1}^{\prime}\right)^{2}=m\left(1+p_{3}+\sum_{i=1}^{\left[\frac{m-1}{2}\right]} q_{2 i}\right) w, \\
& u_{2} u_{2}^{\prime}=m \sum_{i=0}^{\left[\frac{m-1}{2}\right]} q_{2 i+1} \cdot w ;
\end{aligned}
$$

- of degree 5:

$$
u_{0} v=0
$$

- of degree 6 :

$$
v^{2}=\left(u_{1}+u_{1}^{\prime}\right) w
$$

\section{REFERENCES}

[1] S. Eilenberg and S. Mac Lane, Cohomology theory in abstract groups. I, Ann. of Math. (2) 48 (1947), 51-78. MR0019092 (8:367f)

[2] H. Cartan and S. Eilenberg, Homological algebra, Princeton Univ. Press, Princeton, NJ, 1999. MR 1731415 (2000h:18022)

[3] M. Gerstenhaber, The cohomology structure of an associative ring, Ann. of Math. (2) 78 (1963), 267-288. MR0161898 (28:5102)

[4] M. Gerstenhaber and S. D. Schack, Algebraic cohomology and deformation theory, Deformation Theory of Algebras and Structures and Applications (Il Ciocco, 1986) (M. Hazewinkel, M. Gerstenhaber, eds.), NATO Adv. Sci. Inst. Ser. C Math. Phys. Sci., vol. 247, Kluwer Acad. Publ., Dordrecht, 1988, pp. 11-264. MR0981619 (90c:16016)

[5] S. F. Siegel and S. J. Witherspoon, The Hochschild cohomology ring of a group algebra, Proc. London Math. Soc. (3) 79 (1999), 131-157. MR.1687539 (2000b:16016)

[6] K. Erdmann and Th. Holm, Twisted bimodules and Hochschild cohomology for self-injective algebras of class $A_{n}$, Forum Math. 11 (1999), 177-201. MR.1680594 (2001c:16018)

[7] A. I. Generalov, Hochschild cohomology of algebras of dihedral type. I: The family $D(3 K)$ in characteristic 2, Algebra i Analiz 16 (2004), no. 6, 53-122; English transl., St. Petersburg Math. J. 16 (2005), no. 6, 961-1012. MR2117449(2005i:16013)

[8] - Hochschild cohomology of algebras of quaternion type, I: Generalized quaternion groups, Algebra i Analiz 18 (2006), no. 1, 55-107; English transl., St. Petersburg Math. J. 18 (2007), no. 1, 37-76. MR2225213(2007d:16021) 
[9] K. Erdmann, Blocks of tame representation type and related algebras, Lecture Notes in Math., vol. 1428, Springer-Verlag, Berlin, 1990. MR 1064107 (91c:20016)

[10] K. Erdmann, Th. Holm, and N. Snashall, Twisted bimodules and Hochschild cohomology for selfinjective algebras of class $A_{n}$. II, Algebr. Represent. Theory 5 (2002), 457-482. MR1935856 (2004a:16013)

[11] M. A. Kachalova, The Hochschild cohomology for the Möbius algebra, Zap. Nauchn. Sem. S.Peterburg. Otdel. Mat. Inst. Steklov. (POMI) 330 (2006), 173-200; English transl., J. Math. Sci. (N. Y.) 140 (2007), no. 5, 699-715. MR2253573 (2007f:16016)

[12] Th. Holm, Hochschild cohomology of tame blocks, J. Algebra 271 (2004), 798-826. MR2025551 (2005c:20019)

[13] T. Hayami, Hochschild cohomology ring of the integral group ring of the generalized quaternion group, SUT J. Math. 38 (2002), no. 1, 83-126. MR1919950 (2003f:16014)

[14] On Hochschild cohomology ring of the integral group ring of the quaternion group, Tsukuba J. Math. 29 (2005), no. 2, 363-387. MR2177018 (2006j:16016)

[15] Th. Holm, The Hochschild cohomology ring of a modular group algebra: The commutative case, Comm. Algebra 24 (1996), 1957-1969. MR1386022 (97c:13012)

[16] C. Cibils and A. Solotar, Hochschild cohomology algebra of abelian groups, Arch. Math. (Basel) 68 (1997), no. 1, 17-21. MR1421841 (97k:13018)

[17] A. I. Generalov, Cohomology of algebras of semidihedral type. I, Algebra i Analiz 13 (2001), no. 4, 54-85; English transl., St. Petersburg Math. J. 13 (2002), no. 4, 549-574. MR1865495(2002h:16020)

[18] D. Handel, On products in the cohomology of the dihedral groups, Tôhoku Math. J. (2) 45 (1993), no. 1, 13-42. MR.1200878 (93m:20072)

[19] H. S. M. Coxeter and W. O. J. Moser, Generators and relations for discrete groups, 3rd ed., Ergeb. Math. Grenzgeb., Bd. 14, Springer-Verlag, New York-Heidelberg, 1972. MR0349820 (50:2313)

Department of Mathematics and Mechanics, St. Petersburg State University, Universitetskil Prospeket 28, Stary ̌ Peterhof, St. Petersburg 198504, Russia

E-mail address: general@pdmi.ras.ru

Received 20/MAR/2007

Translated by THE AUTHOR 